\title{
PETROLOGY AND GEOCHEMISTRY OF UM ESH OPHIOLITE SERPENTINITES, EASTERN DESERT, EGYPT: A NEOPROTEROZOIC METAMORPHOSED SSZ MANTLE
}

\author{
Ayman E Maurice \\ Geology Department, Faculty of Science, Helwan University, 1795 Cairo, Egypt
}

\begin{abstract}
The Um Esh Neoproterozoic ophiolite serpentinites, Eastern Desert of Egypt, comprise massive and foliated varieties which are composed mainly of antigorite with accessory Cr-spinel. Carbonates are variable in abundance and represented by magnesite aggregates and veinlets. The Cr-spinels are usually zoned with fresh cores or relics surrounded by ferritchromite and chromian magnetite rims. The very low $\mathrm{TiO}_{2}$ contents $(<0.025 \mathrm{wt} \%)$ of primary Cr-spinel and the low $\mathrm{Al}_{2} \mathrm{O}_{3} / \mathrm{SiO}_{2}$ values $(0.010-0.016)$ of serpentinite samples are consistent with residual mantle which experienced high degree of partial melting. The $\mathrm{Al}_{2} \mathrm{O}_{3}\left(16-25 \mathrm{wt} \%\right.$ ) and $\mathrm{TiO}_{2}$ (average $=0.007 \mathrm{wt} \%$ ) contents of the primary Cr-spinel overlap those of spinels of suprasubduction zone (SSZ) and mid-oceanic ridge (MOR) peridotites. However, the $\mathrm{Cr} \#$ (0.55-0.66) and Mg\# (0.39-0.59) of the fresh Cr-spinels together with depleted nature of the Um Esh serpentinites are similar to those of modern SSZ fore-arc peridotites. Profiles along zoned Cr-spinels revealed that $\mathrm{Cr}_{2} \mathrm{O}_{3}, \mathrm{Al}_{2} \mathrm{O}_{3}$ and $\mathrm{MgO}$ abundances decreased abruptly, whereas $\mathrm{FeO}$ contents and $\mathrm{Fe \#}$ $\left(\mathrm{Fe}^{3+} / \mathrm{Cr}+\mathrm{Al}+\mathrm{Fe}^{3+}\right)$ increased rapidly from fresh Cr-spinel through ferritchromite to chromian magnetite, with gradual change within the individual metamorphic spinel zones. On the other hand, the variation of $\mathrm{MnO}$ and $\mathrm{NiO}$ abundances is not regular from core to rim. The dominance of antigorite and the presence of metamorphic spinel rims suggest that Um Esh serpentinites have experienced prograde metamorphism up to lower amphibolite facies under oxidizing conditions.
\end{abstract}

Keywords: Neoproterozoic mantle, SSZ ophiolites, serpentinites, Cr-spinel, Eastern Desert.

\section{INTRODUCTION}

Ophiolites are remnants of ancient oceanic lithosphere (upper mantle and crust) obducted into the continental margins. Typical ophiolite sections include residual mantle peridotites and magmatic rocks, which are topped by deep sea sediments known as pelagic sediments. The magmatic rocks comprise layered and isotropic gabbros followed by sheeted dykes and massive and pillowed basalts. According to their tectonic settings, ophiolites are classified into subduction-unrelated and subduction-related types (Dilek and Furnes, 2011, 2014; Pearce, 2014), which differ in the nature of their mantle sections and the composition of their magmatic rocks (Dilek and Furnes, 2014; Pearce, 2014).

The ophiolites of the Arabian-Nubian Shield, including the Egyptian ones, represent remnants of Neoproterozoic oceanic lithosphere, which were tectonically emplaced during closing of Mozambique Ocean existed between East and West Gondwana (Stern, 1994). The ophiolites of Egypt are present in the southern and central segments of the Eastern Desert. On the other hand, the existence of ophiolitic rocks in the North Eastern Desert and Sinai is controversial (Shimron, 1981, 1984; Abdel Khalek et al., 1994; Madbouly, 2000; Takla et al., 2001; Abu El-Enen and Makroum 2003; El Amawy et al., 2004; Mehanna et al., 2004). The ophiolites of Eastern Desert are mostly present as dismembered units, however, complete ophiolite sections were described from Fawakhir in the Central Eastern Desert (e.g. El-Sayed et al., 1999) and Wadi Ghadir in the South Eastern Desert (e.g. El-Sharkawy and El-Bayoumi, 1979). They comprise extensively serpentinized mantle peridotites, Moho transition zone rocks, metagabbros and massive and pillowed metabasalts (e.g. Zimmer et al., 1995; Azer and Stern, 2007; Basta et al., 2011; Gahlan et al., 2012; Ahmed, 2013; Khedr and Arai, 2017). Sheeted dykes and pelagic sediments are 
Maurice, A. E.

occasionally described from some ophiolites (e.g. Fawakhir, Wadi Ghadir and Gerf ophiolites, ElSharkawy and El-Bayoumi, 1979; Basta et al., 2011; Abd El-Rahman et al., 2009; Zimmer et al., 1995).

Dating of Eastern Desert ophiolites indicates Neoproterozoic age ( 730 to $750 \mathrm{Ma}$ ). U-Pb zircon dating of the ophiolites of the Central (Fawakhir) and South (Wadi Gadir, Gerf and Allaqi) Eastern Desert produced similar ages. TIMS U-Pb zircon dating of Fawakhir ophiolitic gabbro gave $736.5 \pm 1.5 \mathrm{Ma}$ (Andresen et al., 2009). Similarly, Allaqi ophiolites were dated at $730 \pm 6$ Ma (Ali et al., 2010) using SHRIMP U-Pb zircon dating of layered ophiolitic gabbro. Kröner et al. (1992) used single grain zircon evaporation technique to date Wadi Ghadir and Gerf ophiolites at $746 \pm 19$ Ma and $741 \pm 21$ Ma, respectively.

Subduction-related and subduction-unrelated settings were proposed for the origin of the Egyptian ophiolites. Generally, these ophiolites are classified as supra-subduction zone types (e.g. Basta et al., 2011; Khedr and Arai, 2017), however, fore-arc (e.g. Azer and Stern, 2007; Abdel-Karim et al., 2016) and back-arc (e.g. Abd El-Rahman et al., 2009; Basta et al., 2011) settings are debated. On the other hand, some Egyptian ophiolites (e.g. Gerf, Zimmer et al., 1995) were considered to belong to Mid-Ocean Ridge (MOR) subduction-unrelated type. Moreover, different settings were proposed for the different rocks of Gerf ophiolite; fore-arc for ultramafic rocks (Abdel-Karim et al., 2016), back-arc for gabbros and major ocean basin for the sheeted dykes and pillow lavas (Zimmer et al., 1995).

This contribution presents mineral chemistry of primary and secondary minerals, and whole rock geochemistry of ophiolitic ultramafic rocks of Um Esh area, Central Eastern Desert, Egypt, aiming to evaluate the nature of a Neoproterozoic mantle and infer the tectonic setting of such ophiolitic rocks. Metamorphism of serpentinites and element mobility during Cr-spinel alteration has been evaluated.

\section{GEOLOGIC SETTING}

Wadi Um Esh area (Fig. 1) is a part of the Wadi Atalla district, which is characterized by NW-trending Atalla shear zone. Wadi Atalla and the surrounding areas represent a western end of the Precambrian rocks of the Central Eastern Desert of Egypt and have been the focus of several geological, geochemical, geochronological and isotopic studies, especially those concerned with the origin of gold mineralization in old gold mines such as Atalla, El-Sid and Fawakhir (e.g. Bakhit, 1994, 2001; Harraz, 1995; Taman, 1996; Zoheir et al., 2018), and fluorite mineralization as in Wadi Um Esh El-Zarga area (Taman, 1996). The Wadi Um Esh area is covered by several units of the Precambrian basement complex of Egypt, which include gneisses and migmatites, serpentinites and related rocks, metagabbro-diorite complex, mafic metavolcanics, metasediments (metamudstones), Hammamat molasse sedimentary rocks and younger granites (Wassef et al., 1973; Taman, 1996). Vein-type fluorite mineralization follows fault zone within the metagabbro-diorite mass (Wassef et al., 1973).

The ophiolitic ultramafic rocks (serpentinites and alteration products) constitute NW-trending belt parallel to Atalla shear zone, extending from Fawakhir granitic pluton in the southeast to Atalla gold mine in the northwest. These ultramafic rocks have structural contacts against the metagabbro-diorite complex, mafic metavolcanics and metasdiments (metamudstones) and are intruded by the younger granites. Akaad and Noweir (1980) proposed that the metagabbro-diorite complex is equivalent to the El Sid ophiolitic metagabbro of the Fawakhir ophiolite. The ophiolitic ultramafic rocks consist of serpentinites and talccarbonates. The serpentinites are fine-grained and comprise massive and foliated varieties. They vary in colour from dark grey to greenish grey or reddish brown and are variably altered to brown foliated talc carbonate rocks, which occasionally have cavernous appearance.

\section{PETROGRAPHY}

The ultramafic rocks of Um Esh are classified into massive and foliated serpentinites and talccarbonates. The serpentinites are fine-grained, composed of interlocking antigorites (Fig. 2A) with accessory Cr-spinel. In the foliated serpentinites, the antigorites are aligned parallel to each other forming schistose texture (Fig. 2B). The Cr-spinel grains are commonly zoned, composed of fresh cores surrounded by ferritchromite and/or chromian magnetite alteration zones (Figs. 2C \& D). The sizes of the 


\section{Petrology and geochemistry of Um Esh ophiolite serpentinites}

different zones vary from grain to another and the fresh Cr-spinel is sometimes present as isolated segments (Fig. 2C) or relics within ferritchromite and/or chromian magnetite. Magnetite occurs as fine disseminations and streaks. Carbonate grains and patches are interstitial to serpentine. They vary in abundance within the same sample and are more abundant in the foliated serpentinite (Fig. 2E). Carbonates are also present as veinlets in the foliated serpentinites. The talc-carbonate rock (Fig. 2F) is composed of talc, carbonates and chlorite with relics of serpentine.

Fig. 1: Location and geologic map of Um Esh area (modified after Wassef et al., 1973), Central Eastern Desert (CED), Egypt. NED: North Eastern Desert.

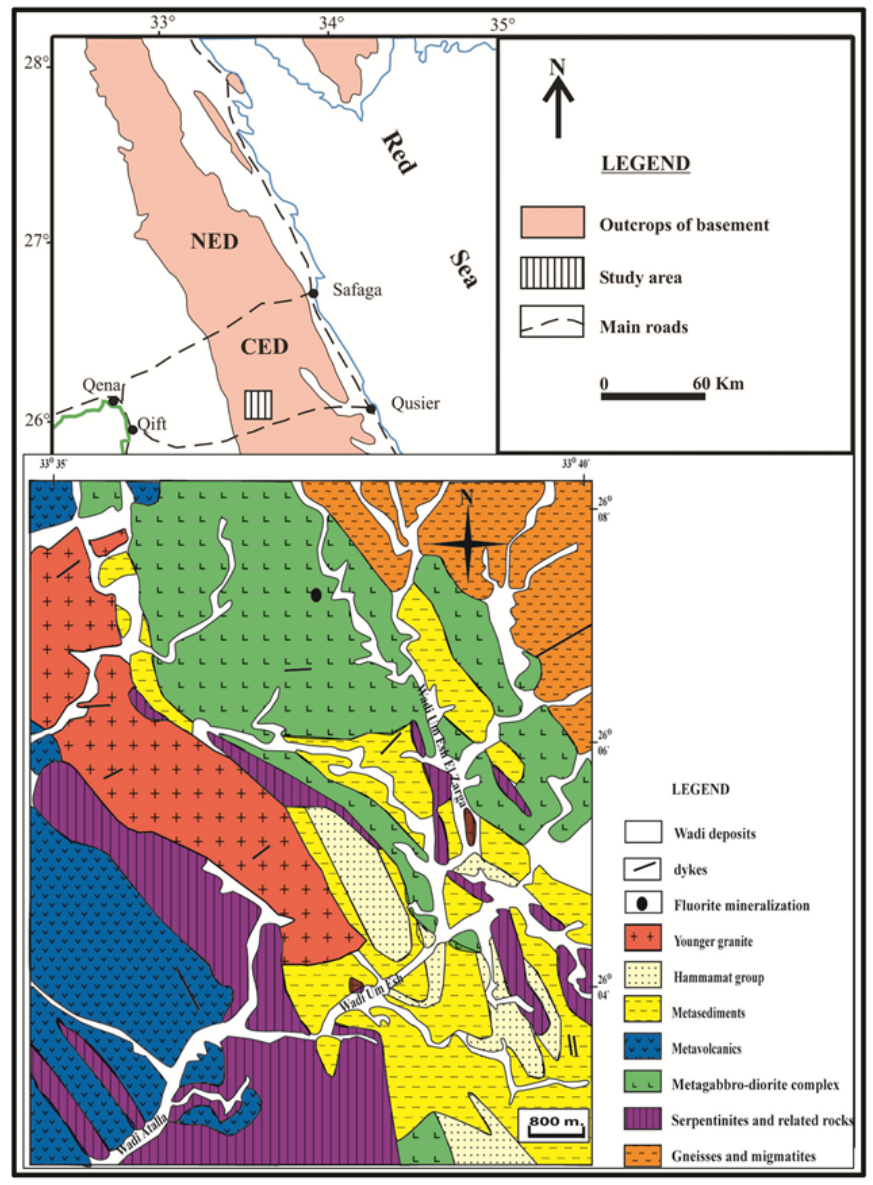

\section{ANALYTICAL TECHNIQUES}

Whole-rock analyses were performed using wave-length dispersive X-ray fluorescence spectrometer (WD-XRF, Axios, PANalytical). Minerals were analyzed using a JEOL JXA-8200 electron probe microanalyzer equipped with five wavelength dispersive spectrometers (WDS). The whole-rock and electron microprobe analyses were carried out at the Institute of Geochemistry and Petrology, ETHZurich, Switzerland. The description and detection limits of the methods used in minerals and whole-rock analyses are given in Basta et al. (2011, 2017).

\section{MINERAL CHEMISTRY}

Unfortunately, the mantle peridotites of Um Esh area were extensively serpentinized and the primary silicate minerals are completely obliterated in the examined samples. However, fresh relics of primary Crspinels survived the serpentinization and metamorphism. Thus, the minerals analyses were restricted to fresh cores and altered zones of $\mathrm{Cr}$-spinel, serpentine minerals and carbonates.

\section{Cr-Spinel}

The Cr-spinel grains of Um Esh serpentinites are usually zoned, with fresh cores or relics surrounded by metamorphic (ferritchromite and chromian magnetite) zones. The microprobe analyses of the different zones either as individual spots or profiles from fresh Cr-spinel to metamorphic spinels are given Tables 1 
Maurice, A. E.

and 2. The fresh cores of Cr-spinel grains of massive and foliated serpentinites have similar composition. On average, the fresh Cr-spinels of massive serpentinites have slightly higher $\mathrm{Cr}_{2} \mathrm{O}_{3}$ (48.22 wt\%), $\mathrm{MgO}$ (10.27 wt\%), $\mathrm{MnO}$ (1.48 wt\%), $\mathrm{Cr} \#$ (61.82) and $\mathrm{Mg \#} \mathrm{(50.64)} \mathrm{compared} \mathrm{with} \mathrm{those} \mathrm{of} \mathrm{the} \mathrm{foliated}$ serpentinites $\left(\mathrm{Cr}_{2} \mathrm{O}_{3}=44.39 \mathrm{wt} \%, \mathrm{MgO}=9.81 \mathrm{wt} \%, \mathrm{MnO}=0.78 \mathrm{wt} \%, \mathrm{Cr} \#=55.25, \mathrm{Mg} \#=46.34\right)$. On the other hand, they have slightly lower $\mathrm{Al}_{2} \mathrm{O}_{3}$ (20.02 wt\%), $\mathrm{FeO}(19.09 \mathrm{wt} \%)$ and $\mathrm{NiO}(0.02 \mathrm{wt} \%)$ values than those of Cr-spinels of the foliated varieties $\left(\mathrm{Al}_{2} \mathrm{O}_{3}=24.13 \mathrm{wt} \%, \mathrm{FeO}=21.15 \mathrm{wt} \%\right.$, $\mathrm{NiO}=0.06$ wt\%). The $\mathrm{Cr}_{2} \mathrm{O}_{3}$ and $\mathrm{Al}_{2} \mathrm{O}_{3}$ abundances of the fresh Cr-spinels are similar to those of mantle spinels and Cr-spinels of the ophiolites of the Arabian-Nubian Shield (Fig. 3).

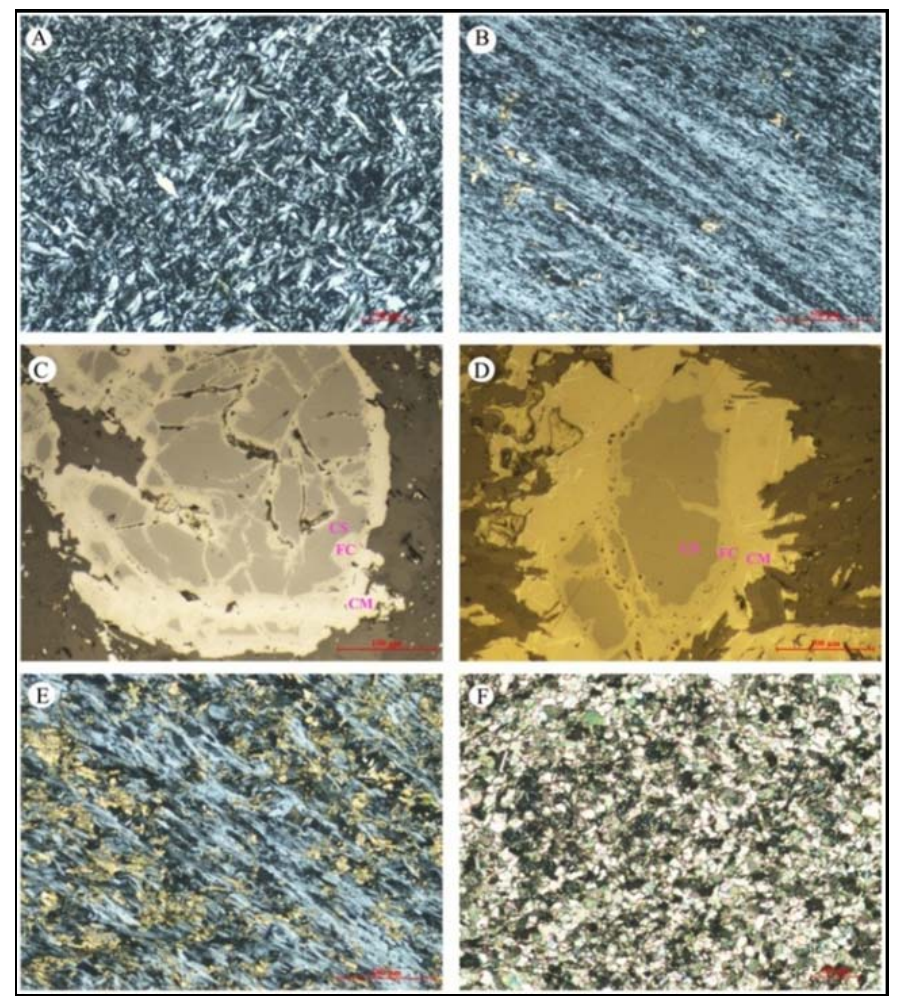

Fig. 2. Petrography of Um Esh serpentinites. (A) Massive antigorite serpentinite, (B) foliated antigorite serpentinite, (C) and (D) Zoned Cr-spinel in massive and foliated serpentinites, respectively, (E) Magnesite aggregates in foliated serpentinite, (F) Talc-carbonate rock with relict serpentine and chlorite. CS, fresh Cr-spinel; FC, ferritchromite; CM, chromian magnetite.

Fig. 3: $\mathrm{Al}_{2} \mathrm{O}_{3}$ versus $\mathrm{Cr}_{2} \mathrm{O}_{3}$ diagram (after Franz and Wirth, 2000) for Cr-spinels of Um Esh serpentinites. Data sources of Cr-spinel of Arabian-Nubian Shield (ANS) ophiolites are Ahmed (2013) and Ahmed et al. (2001, 2012). CED, Central Eastern Desert; SED, South Eastern Desert; AS, Arabian Shield.

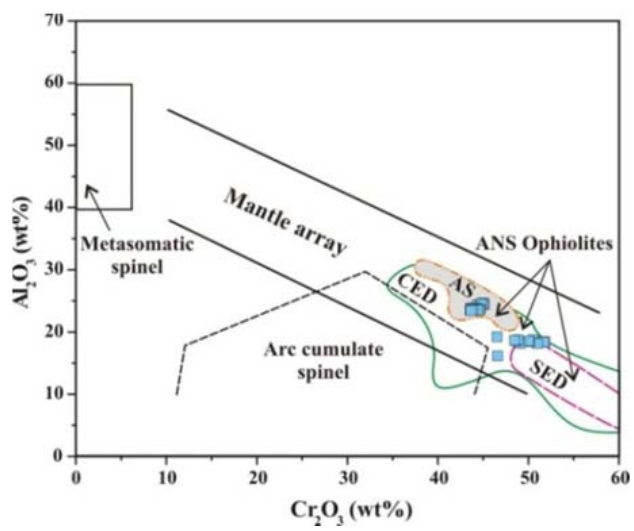

The variation of $\mathrm{Cr}_{2} \mathrm{O}_{3}, \mathrm{Al}_{2} \mathrm{O}_{3}, \mathrm{MgO}, \mathrm{FeO}, \mathrm{MnO}$ and $\mathrm{NiO}$ abundances and $\mathrm{Fe} \#\left(\mathrm{Fe}^{3+} / \mathrm{Cr}+\mathrm{Al}+\mathrm{Fe}^{3+}\right)$ along 4 profiles in $4 \mathrm{Cr}$-spinel grains ( 3 in massive serpentinites and 1 in foliated serpentinites, Table 2) from fresh cores through ferritchromite zones (best developed in profile \#4) to chromian magnetite zones is shown in Figure 4. These profiles reveal that $\mathrm{Al}_{2} \mathrm{O}_{3}$ and $\mathrm{MgO}$ abundances rapidly dropped from the fresh Cr-spinel to the ferritchromite zones then markedly decreased towards the chromian magnetite zones or rims (Fig. 4A and B). The $\mathrm{Cr}_{2} \mathrm{O}_{3}$ contents show slight to moderate decrease from fresh Cr-spinel to the ferritchromite then another significant decrease in the chromian magnetite. Within the individual ferritchromite and chromian magnetite zones the $\mathrm{Cr}_{2} \mathrm{O}_{3}$ contents show gradual decrease. On the other 


\section{Petrology and geochemistry of Um Esh ophiolite serpentinites}

hand, these profiles show that $\mathrm{FeO}$ abundances and Fe\# rapidly increased from the fresh $\mathrm{Cr}$-spinel to the ferritchromite zones then significantly increased again in the chromian magnetite (Fig. 4C and D). Contrary to the other elements, the behaviour of $\mathrm{MnO}$ and $\mathrm{NiO}$ was not uniform (Fig. 4E and F). $\mathrm{MnO}$ contents increase from fresh Cr-spinel to ferritchromite then decrease in the chromian magnetite (Fig. 4E), while the $\mathrm{NiO}$ contents increase from the fresh Cr-spinel through the ferritchromite to the inner zone of chromian magnetite then decrease in the outer rim of chromian magnetite zone (Fig. 4F).

\section{Serpentine}

The composition of serpentines of the Um Esh serpentinites is given in Table 3. The serpentines of the massive and foliated serpentinites have low $\mathrm{Al}_{2} \mathrm{O}_{3}$ abundances (average $=0.62$ and $0.58 \mathrm{wt} \%$, respectively). The serpentine of massive serpentinite has $\mathrm{SiO}_{2}(42.18-44.42 \mathrm{wt} \%$, average $=43.56 \mathrm{wt} \%)$, $\mathrm{MgO}(38.8-41.7 \mathrm{wt} \%$, average $=40.10 \mathrm{wt} \%)$ and $\mathrm{FeO}(1.72-4.18 \mathrm{wt} \%$, average $=2.46 \mathrm{wt} \%)$ abundances which are comparable to those of serpentines of foliated varieties $\left(\mathrm{SiO}_{2}=43.10-44.75 \mathrm{wt} \%\right.$, average $=$ $43.80 \mathrm{wt} \% ; \mathrm{MgO}=37.22-40.55 \mathrm{wt} \%$, average = $39.57 \mathrm{wt} \% ; \mathrm{FeO}=2.49-3.37 \mathrm{wt} \%$, average $=2.85 \mathrm{wt} \%$ ). These serpentines contain some $\mathrm{Cr}\left(\mathrm{Cr}_{2} \mathrm{O}_{3}<0.36 \mathrm{wt} \%\right)$ and $\mathrm{NiO}$ (up to $\left.0.91 \mathrm{wt} \%\right)$. Plotting the analyzed serpentines on the $\mathrm{Al}_{2} \mathrm{O}_{3}$ versus $\mathrm{SiO}_{2}$ diagram (Schwartz et al., 2013) suggests that they are essentially antigorites with few lizardites (Fig. 5). The relatively high $\mathrm{SiO}_{2}$ contents (42.18-44.75 wt\%; average = $43.65 \mathrm{wt} \%)$ of serpentines of Um Esh serpentinites, together with MgO values greater than $37 \mathrm{wt} \%$, indicate that they are antigorites rather than lizardites (Dungan, 1979). XRD results of serpentinite samples from the northwestern tip of this ultramafic belt near Atalla gold mine confirm that the serpentine minerals are dominated by antigorite (Bakhit, 1994).
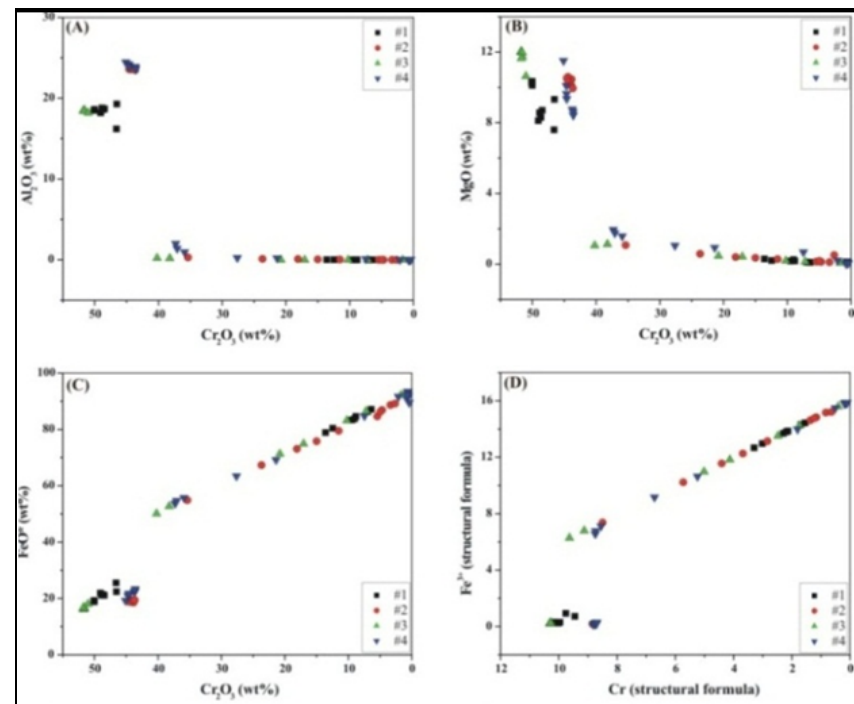

Fig. 4: Profiles along zoned Crspinels showing compositional variations of different zones.
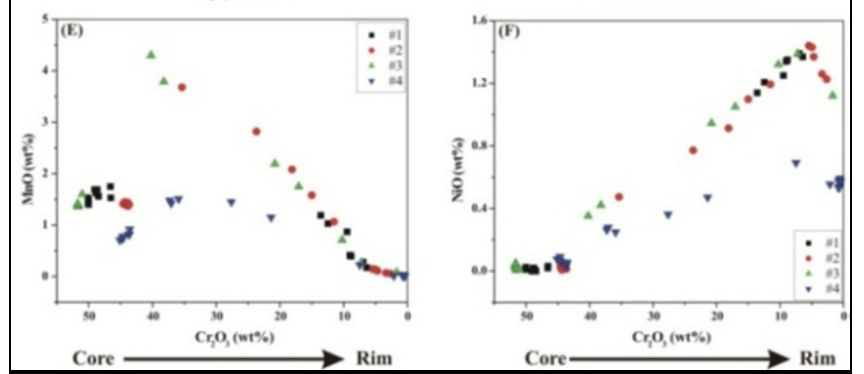

\section{Carbonate}

The microprobe analysis of the carbonates of massive and foliated serpentinites is given in Table 4. The chemical composition of these carbonates reveals that they are magnesites. However, the magnesites of the massive serpentinites have slightly higher $\mathrm{FeO}$ contents (0.89-1.96 wt\%) and lower $\mathrm{CaO}$ (0.04-0.18 wt\%) and $\mathrm{MnO}(0.33-0.43 \mathrm{wt} \%)$ contents compared with those of the foliated serpentinites $(\mathrm{FeO}=1.26-1.52 \mathrm{wt} \%, \mathrm{CaO}=0.23-0.86 \mathrm{wt} \%, \mathrm{MnO}=0.51-0.66 \mathrm{wt} \%)$. 
Maurice, A. E.

Fig. 5. $\mathrm{Al}_{2} \mathrm{O}_{3}$ versus $\mathrm{SiO}_{2}$ diagram (after Schwartz et al., 2013) for the serpentines of Um Esh serpentinites.

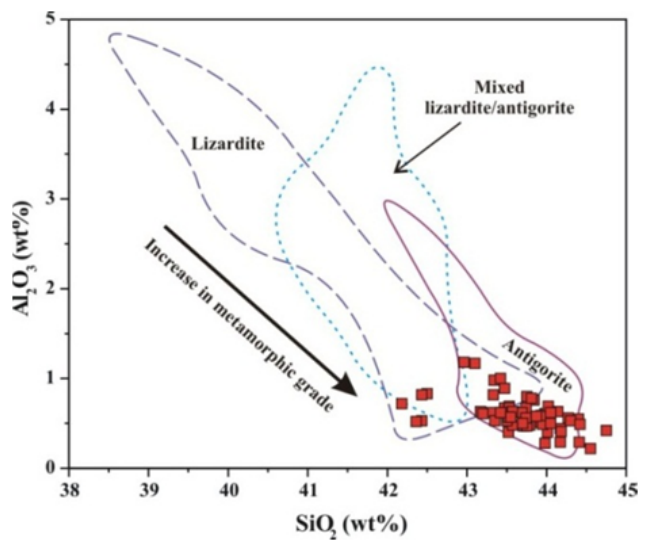

\section{WHOLE-ROCK GEOCHEMISTRY}

Whole-rock chemical composition of 8 massive and foliated serpentinite samples is given in Table 5 . The massive and foliated serpentinites have similar elements abundances (Table 5). The analyzed samples have high $\mathrm{MgO}$ contents (37.06-39.24 wt\%) and $\mathrm{Mg \#} \mathrm{(90.6-91.8),} \mathrm{and} \mathrm{low} \mathrm{Al}_{2} \mathrm{O}_{3}(0.37-0.71 \mathrm{wt} \%)$ and $\mathrm{CaO}$ (0-3.32 wt\%) contents. The generally low $\mathrm{CaO}$ in the analyzed serpentinites reflect low clinopyroxene abundance in the original peridotites. The high $\mathrm{CaO}$ abundances in some samples are attributed to the presence of secondary carbonates. The analyzed samples are largely classified as harzburgites using the normative proportions of olivine, orthopyroxene and clinopyroxene (Fig. 6), suggesting harzburgite protolith. Samples with secondary carbonates plot in the dunite and wehrlite fields. The Um Esh serpentinites have $\mathrm{MgO} / \mathrm{SiO}_{2}$ (0.96-1.11) and low $\mathrm{Al}_{2} \mathrm{O}_{3} / \mathrm{SiO}_{2}$ (0.010-0.016) values comparable to those of fore-arc peridotites (Fig. 7), suggesting that they represent residual mantle rocks which have experienced high degree of partial melting. On average, the analyzed serpentinites have $\mathrm{Ni}$ content $(2540 \mathrm{ppm})$ higher than and $\mathrm{Cr}$ content $(3002 \mathrm{ppm})$ lower than that of primitive mantle $(\mathrm{Ni}=2090$ ppm; $\mathrm{Cr}=3240$ ppm, Hart and Zindler, 1986).

Fig. 6: Nomenclature of the Um Esh serpentinites based on Ol-Opx-Cpx normative composition (after Coleman, 1977).

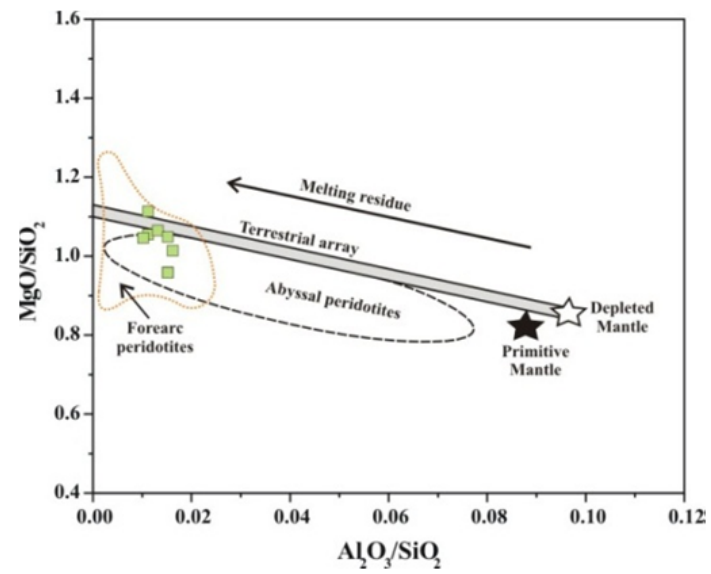

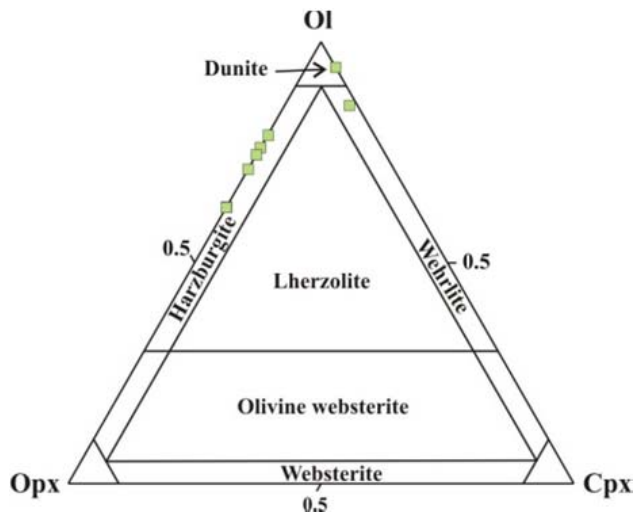

Fig. 7: $\mathrm{MgO} / \mathrm{SiO}_{2}$ versus $\mathrm{Al}_{2} \mathrm{O}_{3} / \mathrm{SiO}_{2}$ diagram for the Um Esh serpentinites. Terrestrial array after Jagoutz et al. (1979) and Hart and Zindler (1986). Abyssal peridotites field after Niu (2004), the forearc peridotites (South Sandwich and IzuBonin-Mariana) field after Pearce et al. (2000) and Parkinson and Pearce (1998). 
Petrology and geochemistry of Um Esh ophiolite serpentinites

Table 1. Representative microprobe analyses of Cr-spinel of Um Esh serpentinites, Eastern Desert, Egypt.

\begin{tabular}{|c|c|c|c|c|c|c|c|c|c|c|c|c|c|c|c|c|c|c|c|}
\hline & \multicolumn{10}{|c|}{ Massive serpentinite } & \multicolumn{9}{|c|}{ Foliated serpentinite } \\
\hline & \multicolumn{6}{|c|}{ Fresh Cr-spinel } & \multicolumn{2}{|c|}{ Altered spinel } & & & \multicolumn{5}{|c|}{ Fresh Cr-spinel } & \multicolumn{4}{|c|}{ Altered spinel } \\
\hline No. & 1 & 2 & 3 & 4 & 5 & 6 & 7 & 8 & 9 & 10 & 11 & 12 & 13 & 14 & 15 & 16 & 17 & 18 & 19 \\
\hline $\mathrm{SiO}_{2}$ & 0.000 & 0.000 & 0.000 & 0.000 & 0.009 & 0.000 & 0.000 & 0.000 & 0.000 & 0.000 & 0.013 & 0.000 & 0.000 & 0.000 & 0.010 & 0.000 & 0.000 & 1.306 & 0.000 \\
\hline $\mathrm{TiO}_{2}$ & 0.002 & 0.001 & 0.000 & 0.000 & 0.001 & 0.000 & 0.003 & 0.000 & 0.006 & 0.013 & 0.013 & 0.010 & 0.016 & 0.000 & 0.000 & 0.010 & 0.016 & 0.016 & 0.000 \\
\hline $\mathrm{Al}_{2} \mathrm{O}_{3}$ & 18.830 & 18.460 & 19.280 & 23.700 & 23.610 & 23.590 & 0.117 & 0.000 & 0.000 & 24.730 & 24.370 & 24.530 & 23.910 & 23.660 & 23.760 & 9.140 & 1.614 & 1.606 & 0.000 \\
\hline $\mathrm{Cr}_{2} \mathrm{O}_{3}$ & 50.520 & 51.790 & 46.540 & 44.630 & 43.910 & 44.340 & 22.990 & 9.290 & 6.580 & 45.020 & 44.650 & 44.820 & 44.250 & 44.040 & 43.970 & 38.980 & 36.580 & 26.780 & 0.563 \\
\hline $\mathrm{FeO}$ & 18.610 & 16.190 & 22.400 & 18.550 & 19.380 & 18.990 & 67.350 & 83.620 & 84.290 & 18.880 & 20.550 & 19.350 & 22.420 & 22.340 & 22.530 & 44.030 & 54.320 & 62.020 & 92.980 \\
\hline $\mathrm{MnO}$ & 1.470 & 1.360 & 1.530 & 1.380 & 1.440 & 1.430 & 2.660 & 0.495 & 0.281 & 0.711 & 0.762 & 0.694 & 0.854 & 0.857 & 0.860 & 1.271 & 1.500 & 1.034 & 0.011 \\
\hline $\mathrm{MgO}$ & 10.330 & 12.030 & 9.320 & 10.800 & 10.120 & 10.350 & 0.564 & 0.267 & 0.175 & 11.650 & 10.340 & 11.500 & 8.760 & 8.630 & 330 & 3.940 & 1.800 & 3.600 & 0.179 \\
\hline $\mathrm{NiO}$ & 0.013 & 0.028 & 0.031 & 0.018 & 0.031 & 0.051 & 0.850 & 1.350 & 1.450 & 0.065 & 0.061 & 0.066 & 0.037 & 0.054 & 0.044 & 0.208 & 0.261 & 0.394 & 0.572 \\
\hline Total & 99.77 & 99.86 & 99.11 & 99.08 & 98.51 & 98.76 & 94.53 & 95.05 & 92.80 & 101.08 & 100.76 & 100.97 & 100.25 & 99.59 & 99.51 & 97.59 & 96.11 & 96.76 & 94.31 \\
\hline & & & & & & & & & & & & & & & & & & & \\
\hline \multicolumn{20}{|c|}{ Formula based on 32 oxygens } \\
\hline $\mathrm{Si}$ & 0.000 & 0.000 & 0.000 & 0.000 & 0.002 & 0.000 & 0.000 & 0.000 & 0.000 & 0.000 & 0.003 & 0.000 & 0.000 & 0.000 & 0.003 & 0.000 & 0.000 & 0.380 & 0.000 \\
\hline $\mathrm{Ti}$ & .000 & 0.000 & 0.000 & 0.000 & & & 0.001 & 0.000 & 0.002 & & & 0.002 & 0.003 & 0.000 & & & 0.004 & 0.003 & 0.000 \\
\hline $\mathrm{Al}$ & 5.645 & 5.477 & 5.836 & 6.983 & 7.025 & 6.995 & 0.042 & 0.000 & 0.000 & 7.100 & 7.085 & 7.061 & 7.066 & 7.046 & 7.091 & 3.028 & 0.569 & 0.551 & 0.000 \\
\hline $\mathrm{Cr}$ & 10.161 & 10.309 & 9.450 & 8.821 & 8.764 & 8.821 & 5.612 & 2.257 & 1.638 & 8.671 & 8.708 & 8.654 & 8.772 & 8.798 & 8.803 & 8.663 & 8.651 & 6.160 & 0.136 \\
\hline $\mathrm{Fe}^{3+}$ & 0.193 & 0.214 & 0.714 & 0.197 & 0.207 & 0.184 & 10.344 & 13.743 & 14.359 & 0.224 & 0.196 & 0.281 & 0.156 & 0.156 & 0.100 & 4.305 & 6.773 & 8.522 & 15.864 \\
\hline $\mathrm{Fe}^{2+}$ & 3.766 & 3.195 & 4.097 & 3.681 & 3.884 & 3.812 & 7.046 & 7.741 & 7.839 & 3.622 & 4.043 & 3.671 & 4.545 & 4.564 & 4.671 & 6.045 & 6.814 & 6.567 & 7.914 \\
\hline $\mathrm{Mn}$ & 0.317 & 0.290 & 0.333 & 0.292 & 0.308 & 0.305 & 0.696 & 0.129 & 0.075 & 0.147 & 0.159 & 0.144 & 0.181 & 0.183 & 0.185 & 0.302 & 0.380 & 0.255 & 0.003 \\
\hline $\mathrm{Mg}$ & 3.918 & 4.515 & 3.569 & 4.025 & 3.809 & 3.883 & 0.259 & 0.122 & 0.082 & 4.231 & 3.803 & 4.187 & 3.275 & 3.251 & 3.145 & 1.651 & 0.803 & 1.562 & 0.082 \\
\hline Total & 24 & 24 & 24 & 24 & 24 & 24 & 24 & 24 & 24 & 24 & 24 & 24 & 24 & 24 & 24 & 24 & 24 & 24 & 24 \\
\hline Cr\# & 0.64 & 0.65 & 0.62 & 0.56 & & & 0.99 & 1.00 & 1.00 & & 0.55 & 0.55 & 0.55 & 0.56 & 0.55 & 0.74 & 0.94 & 0.92 & 1.00 \\
\hline Mg\# & 0.51 & 0.59 & 0.47 & 0.52 & 0.50 & 0.50 & 0.04 & 0.02 & 0.01 & 0.54 & 0.48 & 0.53 & 0.42 & 0.42 & 0.40 & 0.21 & 0.11 & 0.19 & 0.01 \\
\hline $\mathrm{Fe} \#$ & 0.01 & 0.01 & 0.04 & 0.01 & 0.01 & 0.01 & 0.65 & 0.86 & 0.90 & 0.01 & 0.01 & 0.02 & 0.01 & 0.01 & 0.01 & 0.27 & 0.42 & 0.56 & 0.99 \\
\hline
\end{tabular}


Maurice, A. E.

Table 2: Composition and structural formula along 4 traverses in zoned Cr-spinel of Um Esh serpentinites, Eastern Desert, Egypt.

\begin{tabular}{|c|c|c|c|c|c|c|c|c|c|c|c|c|c|c|c|c|c|}
\hline \multicolumn{18}{|c|}{ Massive serpentinite } \\
\hline & \multicolumn{2}{|c|}{ Traverse1 } & & & & & & & & & & & & & & & \\
\hline & Fresh CI & -spinel & \multicolumn{15}{|c|}{ Altered spinel } \\
\hline No. & 1 & 2 & 3 & 4 & 5 & 6 & 7 & 8 & 9 & 10 & 11 & 12 & 13 & 14 & 15 & 16 & 17 \\
\hline $\mathrm{SiO}_{2}$ & 0.000 & 0.000 & 0.000 & 0.000 & 0.000 & 0.000 & 0.000 & 0.446 & 0.000 & 0.000 & 0.000 & 0.013 & 0.000 & 0.026 & 0.000 & 0.000 & 0.000 \\
\hline $\mathrm{TiO}_{2}$ & 0.003 & 0.005 & 0.000 & 0.013 & 0.004 & 0.009 & 0.007 & 0.002 & 0.022 & 0.000 & 0.000 & 0.000 & 0.000 & 0.004 & 0.000 & 0.005 & 0.012 \\
\hline $\mathrm{Al}_{2} \mathrm{O}_{3}$ & 18.530 & 18.510 & 18.500 & 18.610 & 18.190 & 18.590 & 18.790 & 16.210 & 18.700 & 19.280 & 0.000 & 0.000 & 0.000 & 0.026 & 0.018 & 0.000 & 0.000 \\
\hline $\mathrm{Cr}_{2} \mathrm{O}_{3}$ & 50.020 & 50.110 & 50.110 & 50.040 & 49.080 & 48.660 & 48.870 & 46.600 & 48.470 & 46.540 & 9.030 & 6.410 & 8.830 & 13.600 & 12.460 & 6.970 & 9.450 \\
\hline $\mathrm{FeO}$ & 18.830 & 18.990 & 19.030 & 19.310 & 21.900 & 21.360 & 21.160 & 25.550 & 21.110 & 22.400 & 83.760 & 87.140 & 84.630 & 78.890 & 80.420 & 86.350 & 83.390 \\
\hline $\mathrm{MnO}$ & 1.400 & 1.440 & 1.530 & 1.480 & 1.690 & 1.690 & 1.620 & 1.750 & 1.560 & 1.530 & 0.413 & 0.173 & 0.396 & 1.188 & 1.031 & 0.280 & 0.870 \\
\hline $\mathrm{MgO}$ & 10.340 & 10.300 & 10.260 & 10.110 & 8.120 & 8.300 & 8.570 & 7.600 & 8.700 & 9.320 & 0.254 & 0.105 & 0.186 & 0.296 & 0.206 & 0.112 & 0.173 \\
\hline $\mathrm{NiO}$ & 0.020 & 0.011 & 0.010 & 0.025 & 0.000 & 0.012 & 0.020 & 0.019 & 0.000 & 0.031 & 1.340 & 1.370 & 1.350 & 1.139 & 1.207 & 1.390 & 1.250 \\
\hline Total & 99.14 & 99.37 & 99.44 & 99.59 & 98.98 & 98.62 & 99.04 & 98.18 & 98.56 & 99.10 & 94.80 & 95.21 & 95.39 & 95.17 & 95.34 & 95.11 & 95.14 \\
\hline \multicolumn{18}{|c|}{ Formula based on 32 oxygens } \\
\hline Si & 0.000 & 0.000 & 0.000 & 0.000 & 0.000 & 0.000 & 0.000 & 0.118 & 0.000 & 0.000 & 0.000 & 0.004 & 0.000 & 0.008 & 0.000 & 0.000 & 0.000 \\
\hline $\mathrm{Ti}$ & 0.001 & 0.001 & 0.000 & 0.002 & 0.001 & 0.002 & 0.001 & 0.000 & 0.004 & 0.000 & 0.000 & 0.000 & 0.000 & 0.001 & 0.000 & 0.001 & 0.003 \\
\hline $\mathrm{Al}$ & 5.591 & 5.575 & 5.571 & 5.600 & 5.587 & 5.712 & 5.738 & 5.062 & 5.728 & 5.836 & 0.000 & 0.000 & 0.000 & 0.010 & 0.007 & 0.000 & 0.000 \\
\hline $\mathrm{Cr}$ & 10.125 & 10.125 & 10.123 & 10.101 & 10.112 & 10.030 & 10.011 & 9.762 & 9.961 & 9.450 & 2.199 & 1.554 & 2.138 & 3.298 & 3.019 & 1.693 & 2.293 \\
\hline $\mathrm{Fe}^{3+}$ & 0.283 & 0.298 & 0.306 & 0.294 & 0.300 & 0.255 & 0.249 & 0.939 & 0.302 & 0.714 & 13.801 & 14.438 & 13.862 & 12.675 & 12.974 & 14.305 & 13.702 \\
\hline $\mathrm{Fe}^{2+}$ & 3.748 & 3.761 & 3.760 & 3.829 & 4.473 & 4.402 & 4.335 & 4.723 & 4.286 & 4.097 & 7.773 & 7.910 & 7.812 & 7.560 & 7.638 & 7.877 & 7.696 \\
\hline $\mathrm{Mn}$ & 0.304 & 0.312 & 0.331 & 0.320 & 0.373 & 0.373 & 0.355 & 0.393 & 0.343 & 0.333 & 0.108 & 0.045 & 0.103 & 0.309 & 0.268 & 0.073 & 0.226 \\
\hline $\mathrm{Mg}$ & 3.947 & 3.925 & 3.909 & 3.849 & 3.155 & 3.226 & 3.310 & 3.002 & 3.371 & 3.569 & 0.117 & 0.048 & 0.085 & 0.135 & 0.094 & 0.051 & 0.079 \\
\hline Total & 24 & 24 & 24 & 24 & 24 & 24 & 24 & 24 & 24 & 24 & 24 & 24 & 24 & 24 & 24 & 24 & 24 \\
\hline Cr\# & 0.64 & 0.64 & 0.65 & 0.64 & 0.64 & 0.64 & 0.64 & 0.66 & 0.63 & 0.62 & 1.00 & 1.00 & 1.00 & 1.00 & 1.00 & 1.00 & 1.00 \\
\hline Mg\# & 0.51 & 0.51 & 0.51 & 0.50 & 0.41 & 0.42 & 0.43 & 0.39 & 0.44 & 0.47 & 0.01 & 0.01 & 0.01 & 0.02 & 0.01 & 0.01 & 0.01 \\
\hline Fe\# & 0.02 & 0.02 & 0.02 & 0.02 & 0.02 & 0.02 & 0.02 & 0.06 & 0.02 & 0.04 & 0.86 & 0.90 & 0.87 & 0.79 & 0.81 & 0.89 & 0.86 \\
\hline
\end{tabular}


Petrology and geochemistry of Um Esh ophiolite serpentinites

Table 2. Cont.

\begin{tabular}{|c|c|c|c|c|c|c|c|c|c|c|c|c|c|c|c|c|c|}
\hline \multicolumn{18}{|c|}{ Massive serpentinite } \\
\hline & \multicolumn{2}{|c|}{ Traverse2 } & & & & & & & & & & & & & & & \\
\hline & Fresh C & -spinel & \multicolumn{15}{|c|}{ Altered spinel } \\
\hline No. & 1 & 2 & 3 & 4 & 5 & 6 & 7 & 8 & 9 & 10 & 11 & 12 & 13 & 14 & 15 & 16 & 17 \\
\hline $\mathrm{SiO}_{2}$ & 0.000 & 0.014 & 0.034 & 0.007 & 0.000 & 0.009 & 0.009 & 0.030 & 0.000 & 0.000 & 0.053 & 0.000 & 0.007 & 0.000 & 0.003 & 0.000 & 0.226 \\
\hline $\mathrm{TiO}_{2}$ & 0.000 & 0.001 & 0.002 & 0.012 & 0.000 & 0.001 & 0.005 & 0.006 & 0.003 & 0.000 & 0.000 & 0.003 & 0.000 & 0.014 & 0.004 & 0.000 & 0.002 \\
\hline $\mathrm{Al}_{2} \mathrm{O}_{3}$ & 23.590 & 23.620 & 23.580 & 23.590 & 23.590 & 23.610 & 23.490 & 0.291 & 0.108 & 0.092 & 0.050 & 0.032 & 0.000 & 0.000 & 0.000 & 0.000 & 0.000 \\
\hline $\mathrm{Cr}_{2} \mathrm{O}_{3}$ & 44.340 & 43.850 & 44.400 & 44.600 & 44.380 & 43.910 & 43.650 & 35.360 & 23.690 & 18.110 & 15.010 & 11.530 & 4.690 & 5.440 & 4.970 & 3.390 & 2.640 \\
\hline $\mathrm{FeO}$ & 18.990 & 18.520 & 18.770 & 18.880 & 19.000 & 19.380 & 19.410 & 54.870 & 67.370 & 73.110 & 75.850 & 79.500 & 86.910 & 84.660 & 86.300 & 88.670 & 89.210 \\
\hline $\mathrm{MnO}$ & 1.430 & 1.370 & 1.400 & 1.410 & 1.450 & 1.440 & 1.400 & 3.680 & 2.820 & 2.080 & 1.580 & 1.065 & 0.111 & 0.143 & 0.134 & 0.070 & 0.056 \\
\hline $\mathrm{MgO}$ & 10.350 & 10.460 & 10.600 & 10.510 & 10.290 & 10.120 & 9.950 & 1.080 & 0.587 & 0.401 & 0.360 & 0.280 & 0.134 & 0.138 & 0.186 & 0.116 & 0.511 \\
\hline $\mathrm{NiO}$ & 0.051 & 0.033 & 0.009 & 0.027 & 0.008 & 0.031 & 0.014 & 0.474 & 0.772 & 0.913 & 1.098 & 1.194 & 1.370 & 1.440 & 1.430 & 1.260 & 1.226 \\
\hline Total & 98.75 & 97.87 & 98.79 & 99.04 & 98.72 & 98.50 & 97.93 & 95.79 & 95.35 & 94.71 & 94.00 & 93.60 & 93.22 & 91.83 & 93.03 & 93.51 & 93.87 \\
\hline \multirow{2}{*}{\multicolumn{18}{|c|}{ gens }} \\
\hline So & 0000 & $\frac{x y g}{203}$ & $\frac{1 S}{0.008}$ & 0002 & 0,000 & & ל000 & 0009 & 0000 & & & & & & & & \\
\hline $\mathrm{Ti}$ & 000 & 000 & 0.000 & 0.002 & 0.000 & 0.000 & 0.001 & 0.001 & 0.001 & 0.000 & 0.000 & 0.001 & 0.002 & $\begin{array}{l}0.000 \\
0.003\end{array}$ & $\begin{array}{l}0.001 \\
0.001\end{array}$ & 0.000 & 0.001 \\
\hline$\overline{\mathrm{Al}}$ & 995 & 7.049 & 6.976 & 6.970 & 6.998 & 7.025 & 7.034 & 0.104 & 0.039 & 0.034 & 0.018 & 0.012 & 0.000 & 0.000 & 0.000 & 0.000 & 0.000 \\
\hline $\mathrm{Cr}$ & 821 & 8.779 & 8.811 & 8.840 & 8.831 & 8.764 & 8.768 & 8.499 & 5.729 & 4.410 & 3.685 & 2.843 & 1.160 & 1.368 & 1.233 & 0.835 & 0.644 \\
\hline $\mathrm{Fe}^{3+}$ & 0.184 & 0.165 & 0.196 & 0.182 & 0.171 & 0.207 & 0.191 & 7.376 & 10.231 & 11.557 & 12.264 & 13.144 & 14.835 & 14.625 & 14.764 & 15.165 & 15.215 \\
\hline $\mathrm{Fe}^{2+}$ & 3.812 & 3.757 & 3.744 & $\begin{array}{l}3.102 \\
3.776\end{array}$ & 3.828 & 3.884 & 3.933 & 6.574 & $\begin{array}{c}1.201 \\
7.002\end{array}$ & 7.274 & 7.432 & 7.588 & 7.906 & 7.895 & 7.875 & 7.923 & 7.820 \\
\hline $\mathrm{Mn}$ & 0.305 & 0.294 & 0.298 & 0.299 & 0.309 & 0.308 & 0.301 & 0.948 & 0.731 & 0.543 & 0.416 & 0.281 & 0.029 & 0.038 & 0.036 & 0.018 & 0.015 \\
\hline $\mathrm{Mg}$ & 3.883 & 3.949 & 3.967 & 3.928 & 3.861 & 3.809 & 3.769 & 0.490 & 0.268 & 0.184 & 0.167 & 0.130 & 0.062 & 0.065 & 0.087 & 0.054 & 0.235 \\
\hline Total & 24 & 24 & 24 & 24 & 24 & 24 & 24 & 24 & 24 & 24 & 24 & 24 & 24 & 24 & 24 & 24 & 24 \\
\hline $\mathrm{Cr} \#$ & 0.56 & 0.55 & 0.56 & 0.56 & 0.56 & 0.56 & 0.55 & 0.99 & 0.99 & 0.99 & 1.00 & 1.00 & 1.00 & 1.00 & 1.00 & 1.00 & 1.00 \\
\hline Mg\# & 0.50 & 0.51 & 0.51 & 0.51 & 0.50 & 0.50 & 0.49 & 0.07 & 0.04 & 0.02 & 0.02 & 0.02 & 0.01 & 0.01 & 0.01 & 0.01 & 0.03 \\
\hline $\mathrm{Fe} \#$ & 0.01 & 0.01 & 0.01 & 0.01 & 0.01 & 0.01 & 0.01 & 0.46 & 0.64 & 0.72 & 0.77 & 0.82 & 0.93 & 0.91 & 0.92 & 0.95 & 0.96 \\
\hline
\end{tabular}


Maurice, A. E.

Table 2. Cont.

\begin{tabular}{|c|c|c|c|c|c|c|c|c|c|c|c|c|c|c|}
\hline \multicolumn{15}{|c|}{ Massive serpentinite } \\
\hline No. & 1 & 2 & 3 & 4 & 5 & 6 & 7 & 8 & 9 & 10 & 11 & 12 & 13 & 14 \\
\hline $\mathrm{TiO}_{2}$ & 0.000 & 0.000 & 0.020 & 0.001 & 0.000 & 0.000 & 0.001 & 0.006 & 0.002 & 0.014 & 0.000 & 0.005 & 0.015 & 0.000 \\
\hline $\mathrm{Al}_{2} \mathrm{O}_{3}$ & 18.410 & 18.450 & 18.460 & 18.460 & 18.510 & 18.430 & 18.200 & 0.000 & 0.000 & 0.001 & 0.000 & 0.200 & 0.210 & 0.000 \\
\hline $\mathrm{Cr}_{2} \mathrm{O}_{3}$ & 51.560 & 51.730 & 51.640 & 51.790 & 51.590 & 51.710 & 51.010 & 7.190 & 10.250 & 17.050 & 20.790 & 38.220 & 40.210 & 1.680 \\
\hline $\mathrm{MgO}$ & 11.750 & 12.000 & 12.010 & 12.030 & 11.970 & 11.640 & 10.630 & 0.140 & 0.196 & 0.420 & 0.466 & 1.131 & 1.058 & 0.073 \\
\hline $\mathrm{NiO}$ & 0.006 & 0.032 & 0.050 & 0.028 & 0.018 & 0.045 & 0.010 & 1.390 & 1.320 & 1.050 & 0.945 & 0.422 & 0.353 & 1.121 \\
\hline \multirow[t]{2}{*}{ Total } & 99.73 & 99.72 & 99.89 & 99.86 & 99.94 & 100.16 & 99.48 & 95.54 & 95.63 & 95.27 & 95.74 & 96.50 & 96.29 & 95.24 \\
\hline & & & & & & & & & & & & & & \\
\hline \multicolumn{5}{|c|}{ Formula based on 32 oxygens } & & & & & & & & & & \\
\hline $\mathrm{Si}$ & 0.000 & 0.000 & 0.007 & 0.000 & 0.000 & 0.000 & 0.000 & 0.000 & 0.000 & 0.018 & 0.000 & 0.000 & 0.000 & 0.000 \\
\hline $\mathrm{Fe}^{2+}$ & 3.281 & 3.196 & 3.204 & 3.195 & 3.211 & 3.326 & 3.609 & 7.864 & 7.724 & 7.371 & 7.222 & 6.520 & 6.422 & 7.947 \\
\hline $\mathrm{Mn}$ & 0.295 & 0.293 & 0.296 & 0.290 & 0.296 & 0.303 & 0.346 & 0.070 & 0.184 & 0.454 & 0.566 & 0.970 & 1.104 & 0.020 \\
\hline $\mathrm{Mg}$ & 4.423 & 4.511 & 4.507 & 4.515 & 4.489 & 4.370 & 4.043 & 0.064 & 0.089 & 0.192 & 0.212 & 0.509 & 0.478 & 0.033 \\
\hline Total & 24 & 24 & 24 & 24 & 24 & 24 & 24 & 24 & 24 & 24 & 24 & 24 & 24 & 24 \\
\hline Cr\# & 0.65 & 0.65 & 0.65 & 0.65 & 0.65 & 0.65 & 0.65 & 1.00 & 1.00 & 1.00 & 1.00 & 0.99 & 0.99 & 1.00 \\
\hline Mg\# & 0.57 & 0.59 & 0.58 & 0.59 & 0.58 & 0.57 & 0.53 & 0.01 & 0.01 & 0.03 & 0.03 & 0.07 & 0.07 & 0.00 \\
\hline Fe\# & 0.01 & 0.01 & 0.01 & 0.01 & 0.02 & 0.01 & 0.01 & 0.89 & 0.85 & 0.74 & 0.69 & 0.43 & 0.39 & 0.97 \\
\hline
\end{tabular}


Petrology and geochemistry of Um Esh ophiolite serpentinites

Table 2. Cont.

\begin{tabular}{|c|c|c|c|c|c|c|c|c|c|c|c|c|c|c|c|c|c|c|c|c|c|}
\hline \multicolumn{22}{|c|}{ Foliated serpentinite } \\
\hline & \multirow{2}{*}{\multicolumn{3}{|c|}{$\begin{array}{l}\text { Traverse4 } \\
\text { Fresh Cr-spinel }\end{array}$}} & & & & & & & & & & & & & & & & & & \\
\hline & & & & & & & & \multicolumn{14}{|c|}{ Altered spinel } \\
\hline No. & 1 & 2 & 3 & 4 & 5 & 6 & 7 & 8 & 9 & 10 & 11 & 12 & 13 & 14 & 15 & 16 & 17 & 18 & 19 & 20 & 21 \\
\hline $\mathrm{SiO}_{2}$ & 0.009 & 0.000 & 0.000 & 0.000 & 0.000 & 0.014 & 0.000 & 0.012 & 0.003 & 0.000 & 0.047 & 0.061 & 0.282 & 0.000 & 0.001 & 0.000 & 0.000 & 0.018 & 0.000 & 0.000 & 0.052 \\
\hline $\mathrm{TiO}_{2}$ & 0.013 & 0.009 & 0.005 & 0.024 & 0.004 & 0.015 & 0.018 & 0.007 & 0.019 & 0.008 & 0.018 & 0.009 & 0.007 & 0.000 & 0.006 & 0.000 & 0.000 & 0.003 & 0.000 & 0.014 & 0.000 \\
\hline $\mathrm{Al}_{2} \mathrm{O}_{3}$ & 24.450 & 24.210 & 23.980 & 23.890 & 23.890 & 23.850 & 23.580 & 1.980 & 1.389 & 0.945 & 0.229 & 0.199 & 0.095 & 0.000 & 0.000 & 0.000 & 0.000 & 0.011 & 0.000 & 0.000 & 0.000 \\
\hline $\mathrm{Cr}_{2} \mathrm{O}_{3}$ & 45.100 & 44.660 & 44.620 & 44.600 & 43.710 & 43.590 & 43.560 & 37.290 & 37.050 & 35.900 & 27.620 & 21.400 & 7.470 & 2.130 & 0.680 & 0.573 & 0.592 & 0.521 & 0.759 & 0.784 & 0.402 \\
\hline $\mathrm{FeO}$ & 19.200 & 20.730 & 20.970 & 21.730 & 22.410 & 22.500 & 23.330 & 53.710 & 54.640 & 55.680 & 63.450 & 69.170 & 84.750 & 91.630 & 93.340 & 93.460 & 92.710 & 91.730 & 90.780 & 92.320 & 89.460 \\
\hline $\mathrm{MnO}$ & 0.705 & 0.745 & 0.735 & 0.781 & 0.824 & 0.820 & 0.916 & 1.480 & 1.420 & 1.510 & 1.450 & 1.150 & 0.224 & 0.009 & 0.018 & 0.028 & 0.005 & 0.033 & 0.029 & 0.018 & 0.007 \\
\hline $\mathrm{MgO}$ & 11.510 & 10.090 & 9.630 & 9.350 & 8.760 & 8.610 & 8.420 & 1.940 & 1.760 & 1.573 & 1.048 & 0.935 & 0.680 & 0.206 & 0.162 & 0.138 & 0.117 & 0.087 & 0.024 & 0.031 & 0.042 \\
\hline $\mathrm{NiO}$ & 0.075 & 0.092 & 0.066 & 0.065 & 0.028 & 0.047 & 0.056 & 0.263 & 0.278 & 0.248 & 0.362 & 0.471 & 0.692 & 0.555 & 0.533 & 0.581 & 0.540 & 0.592 & 0.574 & 0.532 & 0.571 \\
\hline Total & 101.06 & 100.54 & 100.01 & 100.44 & 99.63 & 99.45 & 99.88 & 96.68 & 96.56 & 95.86 & 94.22 & 93.39 & 94.20 & 94.53 & 94.74 & 94.78 & 93.96 & 92.99 & 92.17 & 93.70 & 90.53 \\
\hline & & & & & & & & & & & & & & & & & & & & & \\
\hline \multicolumn{22}{|c|}{ Formula based on 32 oxygens } \\
\hline Si & 0.002 & 0.000 & 0.000 & 0.000 & 0.000 & 0.003 & 0.000 & 0.003 & 0.001 & 0.000 & 0.014 & 0.019 & 0.086 & 0.000 & 0.000 & 0.000 & 0.000 & 0.006 & 0.000 & 0.000 & 0.017 \\
\hline $\mathrm{Ti}$ & 0.002 & 0.002 & 0.001 & 0.004 & 0.001 & 0.003 & 0.003 & 0.001 & 0.004 & 0.002 & 0.004 & 0.002 & 0.002 & 0.000 & 0.001 & 0.000 & 0.000 & 0.001 & 0.000 & 0.003 & 0.000 \\
\hline $\mathrm{Al}$ & 7.035 & 7.069 & 7.061 & 7.023 & 7.097 & 7.105 & 7.014 & 0.692 & 0.488 & 0.335 & 0.083 & 0.073 & 0.034 & 0.000 & 0.000 & 0.000 & 0.000 & 0.004 & 0.000 & 0.000 & 0.000 \\
\hline $\mathrm{Cr}$ & 8.705 & 8.748 & 8.813 & 8.796 & 8.711 & 8.712 & 8.692 & 8.746 & 8.736 & 8.547 & 6.716 & 5.243 & 1.808 & 0.514 & 0.164 & 0.138 & 0.144 & 0.128 & 0.188 & 0.191 & 0.101 \\
\hline $\mathrm{Fe}^{3+}$ & 0.251 & 0.180 & 0.124 & 0.172 & 0.191 & 0.170 & 0.287 & 6.551 & 6.766 & 7.114 & 9.164 & 10.642 & 13.981 & 15.486 & 15.833 & 15.862 & 15.856 & 15.855 & 15.812 & 15.803 & 15.865 \\
\hline $\mathrm{Fe}^{2+}$ & 3.668 & 4.115 & 4.257 & 4.361 & 4.533 & 4.586 & 4.637 & 6.773 & 6.860 & 6.908 & 7.154 & 7.283 & 7.719 & 7.902 & 7.924 & 7.926 & 7.944 & 7.957 & 7.977 & 7.979 & 7.986 \\
\hline $\mathrm{Mn}$ & 0.146 & 0.156 & 0.156 & 0.165 & 0.176 & 0.175 & 0.196 & 0.372 & 0.359 & 0.385 & 0.378 & 0.302 & 0.058 & 0.002 & 0.005 & 0.007 & 0.001 & 0.009 & 0.008 & 0.005 & 0.002 \\
\hline $\mathrm{Mg}$ & 4.189 & 3.727 & 3.587 & 3.477 & 3.292 & 3.245 & 3.168 & 0.858 & 0.783 & 0.706 & 0.480 & 0.432 & 0.311 & 0.094 & 0.073 & 0.063 & 0.053 & 0.040 & 0.011 & 0.014 & 0.020 \\
\hline Total & 24 & 24 & 24 & 24 & 24 & 24 & 24 & 24 & 24 & 24 & 24 & 24 & 24 & 24 & 24 & 24 & 24 & 24 & 24 & 24 & 24 \\
\hline $\mathrm{Cr} \#$ & 0.55 & 0.55 & 0.56 & 0.56 & 0.55 & 0.55 & 0.55 & 0.93 & 0.95 & 0.96 & 0.99 & 0.99 & 0.98 & 1.00 & 1.00 & 1.00 & 1.00 & 0.97 & 1.00 & 1.00 & 1.00 \\
\hline Mg\# & 0.53 & 0.48 & 0.46 & 0.44 & 0.42 & 0.41 & 0.41 & 0.11 & 0.10 & 0.09 & 0.06 & 0.06 & 0.04 & 0.01 & 0.01 & 0.01 & 0.01 & 0.01 & 0.00 & 0.00 & 0.00 \\
\hline Fe\# & 0.02 & 0.01 & 0.01 & 0.01 & 0.01 & 0.01 & 0.02 & 0.41 & 0.42 & 0.44 & 0.57 & 0.67 & 0.88 & 0.97 & 0.99 & 0.99 & 0.99 & 0.99 & 0.99 & 0.99 & 0.99 \\
\hline
\end{tabular}


Maurice, A. E.

Table 3. Composition and structural formula of serpentines of Um Esh serpentinites, Eastern Desert, Egypt.

\begin{tabular}{|c|c|c|c|c|c|c|c|c|c|c|c|c|c|c|c|c|c|c|c|c|c|}
\hline & \multicolumn{21}{|c|}{ Massive serpentinite } \\
\hline No. & 1 & 2 & 3 & 4 & 5 & 6 & 7 & 8 & 9 & 10 & 11 & 12 & 13 & 14 & 15 & 16 & 17 & 18 & 19 & 20 & 21 \\
\hline $\mathrm{SiO}_{2}$ & 43.420 & 43.710 & 43.170 & 42.430 & 43.440 & 44.170 & 43.500 & 42.960 & 42.360 & 43.350 & 43.720 & 43.750 & 43.950 & 43.930 & 44.020 & 44.140 & 43.690 & 44.050 & 44.400 & 43.570 & 43.520 \\
\hline $\mathrm{TiO}_{2}$ & 0.000 & 0.000 & 0.000 & 0.000 & 0.000 & 0.000 & 0.000 & 0.011 & 0.000 & 0.000 & 0.000 & 0.000 & 0.000 & 0.000 & 0.000 & 0.000 & 0.000 & 0.000 & 0.000 & 0.000 & 0.000 \\
\hline $\mathrm{Al}_{2} \mathrm{O}_{3}$ & 1.004 & 0.465 & 0.632 & 0.530 & 0.615 & 0.290 & 0.529 & 1.179 & 0.520 & 0.531 & 0.502 & 0.466 & 0.492 & 0.590 & 0.686 & 0.632 & 0.620 & 0.619 & 0.551 & 0.634 & 0.570 \\
\hline $\mathrm{FeO}$ & 2.290 & 1.970 & 2.510 & 2.290 & 2.130 & 1.720 & 2.060 & 2.430 & 3.810 & 2.210 & 2.000 & 1.810 & 2.020 & 2.330 & 2.450 & 2.720 & 2.840 & 2.870 & 2.050 & 2.120 & 2.090 \\
\hline $\mathrm{MnO}$ & 0.016 & 0.042 & 0.038 & 0.052 & 0.034 & 0.043 & 0.023 & 0.035 & 0.054 & 0.030 & 0.028 & 0.053 & 0.032 & 0.054 & 0.059 & 0.047 & 0.055 & 0.034 & 0.029 & 0.050 & 0.052 \\
\hline $\mathrm{MgO}$ & 40.260 & 40.310 & 39.550 & 40.320 & 40.230 & 40.780 & 39.900 & 40.130 & 39.200 & 39.770 & 40.970 & 41.130 & 40.830 & 40.070 & 39.410 & 39.320 & 39.380 & 40.030 & 41.700 & 40.440 & 40.500 \\
\hline $\mathrm{CaO}$ & 0.006 & 0.012 & 0.000 & 0.013 & 0.000 & 0.013 & 0.000 & 0.000 & 0.014 & 0.011 & 0.000 & 0.005 & 0.000 & 0.000 & 0.011 & 0.007 & 0.019 & 0.000 & 0.003 & 0.007 & 0.005 \\
\hline $\mathrm{Na}_{2} \mathrm{O}$ & 0.000 & 0.000 & 0.000 & 0.000 & 0.013 & 0.000 & 0.000 & 0.020 & 0.017 & 0.000 & 0.000 & 0.002 & 0.000 & 0.000 & 0.009 & 0.000 & 0.000 & 0.000 & 0.000 & 0.000 & 0.000 \\
\hline $\mathrm{K}_{2} \mathrm{O}$ & 0.001 & 0.000 & 0.006 & 0.008 & 0.004 & 0.000 & 0.005 & 0.008 & 0.012 & 0.001 & 0.000 & 0.000 & 0.000 & 0.000 & 0.008 & 0.004 & 0.003 & 0.006 & 0.005 & 0.000 & 0.000 \\
\hline $\mathrm{Cr}_{2} \mathrm{O}_{3}$ & 0.030 & 0.121 & 0.121 & 0.091 & 0.094 & 0.005 & 0.078 & 0.273 & 0.243 & 0.116 & 0.088 & 0.093 & 0.104 & 0.112 & 0.111 & 0.089 & 0.095 & 0.119 & 0.124 & 0.113 & 0.061 \\
\hline $\mathrm{NiO}$ & 0.093 & 0.278 & 0.115 & 0.162 & 0.050 & 0.042 & 0.325 & 0.157 & 0.404 & 0.265 & 0.114 & 0.086 & 0.113 & 0.101 & 0.128 & 0.261 & 0.268 & 0.283 & 0.053 & 0.111 & 0.165 \\
\hline Total & 87.12 & 86.91 & 86.14 & 85.89 & 86.61 & 87.06 & 86.42 & 87.20 & 86.63 & 86.28 & 87.42 & 87.39 & 87.54 & 87.19 & 86.89 & 87.22 & 86.97 & 88.01 & 88.91 & 87.04 & 86.96 \\
\hline \multicolumn{22}{|c|}{ Formula based on 9 oxygens } \\
\hline $\mathrm{Si}$ & 2.593 & 2.616 & 2.611 & 2.578 & 2.608 & 2.630 & 2.619 & 2.571 & 2.577 & 2.616 & 2.601 & 2.601 & 2.610 & 2.621 & 2.635 & 2.637 & 2.622 & 2.614 & 2.597 & 2.604 & 2.604 \\
\hline $\mathrm{Al}$ & 0.071 & 0.033 & 0.045 & 0.038 & 0.044 & 0.020 & 0.037 & 0.083 & 0.037 & 0.038 & 0.035 & 0.033 & 0.034 & 0.041 & 0.048 & 0.045 & 0.044 & 0.043 & 0.038 & 0.045 & 0.040 \\
\hline $\mathrm{Ti}$ & 0.000 & 0.000 & 0.000 & 0.000 & 0.000 & 0.000 & 0.000 & 0.001 & 0.000 & 0.000 & 0.000 & 0.000 & 0.000 & 0.000 & 0.000 & 0.000 & 0.000 & 0.000 & 0.000 & 0.000 & 0.000 \\
\hline $\mathrm{Fe}$ & 0.114 & 0.099 & 0.127 & 0.116 & 0.107 & 0.086 & 0.104 & 0.122 & 0.194 & 0.112 & 0.100 & 0.090 & 0.100 & 0.116 & 0.123 & 0.136 & 0.143 & 0.142 & 0.100 & 0.106 & 0.105 \\
\hline $\mathrm{Mn}$ & 0.001 & 0.002 & 0.002 & 0.003 & 0.002 & 0.002 & 0.001 & 0.002 & 0.003 & 0.002 & 0.001 & 0.003 & 0.002 & 0.003 & 0.003 & 0.002 & 0.003 & 0.002 & 0.001 & 0.003 & 0.003 \\
\hline $\mathrm{Mg}$ & 3.585 & 3.596 & 3.566 & 3.652 & 3.600 & 3.619 & 3.580 & 3.580 & 3.554 & 3.577 & 3.633 & 3.645 & 3.614 & 3.564 & 3.516 & 3.502 & 3.523 & 3.541 & 3.636 & 3.603 & 3.612 \\
\hline $\mathrm{Ca}$ & 0.000 & 0.001 & 0.000 & 0.001 & 0.000 & 0.001 & 0.000 & 0.000 & 0.001 & 0.001 & 0.000 & 0.000 & 0.000 & 0.000 & 0.001 & 0.000 & 0.001 & 0.000 & 0.000 & 0.000 & 0.000 \\
\hline $\mathrm{Na}$ & 0.000 & 0.000 & 0.000 & 0.000 & 0.002 & 0.000 & 0.000 & 0.002 & 0.002 & 0.000 & 0.000 & 0.000 & 0.000 & 0.000 & 0.001 & 0.000 & 0.000 & 0.000 & 0.000 & 0.000 & 0.000 \\
\hline $\mathrm{K}$ & 0.000 & 0.000 & 0.000 & 0.001 & 0.000 & 0.000 & 0.000 & 0.001 & 0.001 & 0.000 & 0.000 & 0.000 & 0.000 & 0.000 & 0.001 & 0.000 & 0.000 & 0.000 & 0.000 & 0.000 & 0.000 \\
\hline $\mathrm{Cr}$ & 0.001 & 0.006 & 0.006 & 0.004 & 0.004 & 0.000 & 0.004 & 0.013 & 0.012 & 0.006 & 0.004 & 0.004 & 0.005 & 0.005 & 0.005 & 0.004 & 0.004 & 0.006 & 0.006 & 0.005 & 0.003 \\
\hline $\mathrm{Ni}$ & 0.004 & 0.013 & 0.006 & 0.008 & 0.002 & 0.002 & 0.016 & 0.008 & 0.020 & 0.013 & 0.005 & 0.004 & 0.005 & 0.005 & 0.006 & 0.013 & 0.013 & 0.014 & 0.002 & 0.005 & 0.008 \\
\hline Total & 6.370 & 6.365 & 6.363 & 6.401 & 6.369 & 6.360 & 6.361 & 6.382 & 6.400 & 6.363 & 6.380 & 6.381 & 6.371 & 6.356 & 6.339 & 6.339 & 6.354 & 6.362 & 6.381 & 6.371 & 6.375 \\
\hline
\end{tabular}


Petrology and geochemistry of Um Esh ophiolite serpentinites

Table 3. Cont.

\begin{tabular}{|c|c|c|c|c|c|c|c|c|c|c|c|c|c|c|c|c|c|c|c|c|c|}
\hline \multicolumn{16}{|c|}{ Massive serpentinite } & \multicolumn{6}{|c|}{ Foliated serpentinite } \\
\hline No. & 22 & 23 & 24 & 25 & 26 & 27 & 28 & 29 & 30 & 31 & 32 & 33 & 34 & 35 & 36 & 37 & 38 & 39 & 40 & 41 & 42 \\
\hline $\mathrm{SiO}_{2}$ & 43.740 & 43.550 & 43.420 & 43.700 & 42.180 & 42.500 & 42.430 & 43.330 & 43.810 & 43.200 & 44.050 & 44.270 & 43.870 & 44.300 & 44.420 & 43.470 & 43.520 & 44.170 & 43.790 & 44.410 & 43.940 \\
\hline $\mathrm{TiO}_{2}$ & 0.000 & 0.002 & 0.000 & 0.000 & 0.006 & 0.000 & 0.000 & 0.000 & 0.000 & 0.000 & 0.000 & 0.000 & 0.000 & 0.004 & 0.000 & 0.000 & 0.000 & 0.000 & 0.000 & 0.000 & 0.000 \\
\hline $\mathrm{Al}_{2} \mathrm{O}_{3}$ & 0.562 & 0.571 & 0.616 & 0.502 & 0.721 & 0.830 & 0.816 & 0.821 & 0.777 & 0.607 & 0.502 & 0.559 & 0.583 & 0.532 & 0.486 & 0.890 & 0.402 & 0.440 & 0.484 & 0.294 & 0.549 \\
\hline $\mathrm{FeO}$ & 2.010 & 2.250 & 3.060 & 1.920 & 3.880 & 4.180 & 3.970 & 2.370 & 2.480 & 2.860 & 2.530 & 2.070 & 2.090 & 2.160 & 2.080 & 2.790 & 2.620 & 2.570 & 2.710 & 2.590 & 3.030 \\
\hline $\mathrm{MnO}$ & 0.032 & 0.042 & 0.067 & 0.016 & 0.050 & 0.058 & 0.042 & 0.058 & 0.050 & 0.046 & 0.039 & 0.048 & 0.046 & 0.020 & 0.044 & 0.069 & 0.050 & 0.026 & 0.029 & 0.050 & 0.038 \\
\hline $\mathrm{MgO}$ & 40.300 & 40.350 & 39.520 & 40.310 & 38.800 & 39.160 & 39.180 & 39.830 & 40.230 & 40.010 & 40.290 & 40.830 & 40.420 & 40.070 & 39.980 & 40.130 & 40.550 & 39.710 & 39.530 & 38.760 & 39.830 \\
\hline $\mathrm{CaO}$ & 0.000 & 0.000 & 0.003 & 0.009 & 0.016 & 0.018 & 0.009 & 0.004 & 0.003 & 0.005 & 0.006 & 0.008 & 0.019 & 0.004 & 0.009 & 0.014 & 0.015 & 0.014 & 0.022 & 0.015 & 0.023 \\
\hline $\mathrm{Na}_{2} \mathrm{O}$ & 0.000 & 0.009 & 0.000 & 0.000 & 0.046 & 0.009 & 0.010 & 0.010 & 0.000 & 0.008 & 0.000 & 0.031 & 0.000 & 0.003 & 0.005 & 0.000 & 0.000 & 0.000 & 0.000 & 0.000 & 0.000 \\
\hline $\mathrm{K}_{2} \mathrm{O}$ & 0.002 & 000 & 005 & 0.000 & 0.025 & 0.022 & 0.013 & 0.015 & 0.006 & 0.004 & 0.002 & 0.008 & 0.009 & 0.006 & 0.005 & 0.002 & 0.000 & 0.002 & 0.004 & 0.000 & 0.004 \\
\hline $\mathrm{Cr}_{2} \mathrm{O}_{3}$ & 0.071 & 0.131 & 0.179 & 0.134 & 0.263 & 0.302 & 0.188 & 0.288 & 0.260 & 0.230 & 0.215 & 0.152 & 0.223 & 0.263 & 0.206 & 0.048 & 0.162 & 0.033 & 0.086 & 0.031 & 0.085 \\
\hline $\mathrm{NiO}$ & 0.151 & 0.253 & 0.337 & 0.072 & 0.419 & 0.497 & 0.313 & 0.165 & $\begin{array}{l}0.187 \\
\end{array}$ & 0.275 & 0.213 & 0.109 & 0.194 & 0.230 & 0.204 & 0.178 & 0.145 & 0.373 & 0.177 & 0.244 & 0.385 \\
\hline Total & 86.87 & 87.16 & 87.21 & 86.66 & 86.41 & 87.57 & 86.97 & 86.89 & 87.80 & 87.25 & 87.85 & 88.09 & 87.45 & 87.59 & 87.44 & 87.59 & 87.46 & 87.34 & 86.83 & 86.39 & 87.88 \\
\hline \multicolumn{22}{|c|}{ oxygens } \\
\hline $\mathrm{Si}$ & 2.616 & 2.603 & 2.606 & 2.618 & 2.574 & 2.564 & 2.570 & 2.600 & 2.602 & 2.591 & 2.615 & 2.613 & 2.610 & 2.630 & 2.639 & 2.592 & 2.598 & 2.635 & 2.629 & 2.672 & 2.615 \\
\hline $\mathrm{Al}$ & 0.040 & 0.040 & 0.044 & 0.035 & 0.052 & 0.059 & 0.058 & 0.058 & 0.054 & 0.043 & 0.035 & 0.039 & 0.041 & 0.037 & 0.034 & 0.063 & 0.028 & 0.031 & 0.034 & 0.021 & 0.039 \\
\hline $\mathrm{Ti}$ & 0.000 & 0.000 & 0.000 & 0.000 & 0.000 & 0.000 & 0.000 & 0.000 & 0.000 & 0.000 & 0.000 & 0.000 & 0.000 & 0.000 & 0.000 & 0.000 & 0.000 & 0.000 & 0.000 & 0.000 & 0.000 \\
\hline $\mathrm{Fe}$ & 0.101 & 0.112 & 0.154 & 0.096 & 0.198 & 0.211 & 0.201 & 0.119 & 0.123 & 0.143 & 0.126 & 0.102 & 0.104 & 0.107 & 0.103 & 0.139 & 0.131 & 0.128 & 0.136 & 0.130 & 0.151 \\
\hline Mn & .002 & 0.002 & 0.003 & 0.001 & 0.003 & 0.003 & 0.002 & 0.003 & 0.002 & 0.002 & 0.002 & 0.002 & 0.002 & 0.001 & 0.002 & 0.003 & 0.003 & & 0.001 & 0.003 & 0.002 \\
\hline $\mathrm{Mg}$ & 3.593 & 3.596 & 3.536 & 3.600 & 3.529 & 3.522 & 3.538 & 3.562 & 3.562 & 3.577 & 3.565 & 3.593 & 3.585 & 3.546 & 3.540 & 3.567 & 3.609 & 3.532 & 3.537 & 3.477 & 3.534 \\
\hline $\mathrm{Ca}$ & 0.000 & 0.000 & 0.000 & 0.001 & 0.001 & 0.001 & 0.001 & 0.000 & 0.000 & 0.000 & 0.000 & 0.001 & 0.001 & 0.000 & 0.001 & 0.001 & 0.001 & 0.001 & 0.001 & 0.001 & 0.001 \\
\hline $\mathrm{Na}$ & 0.000 & 0.001 & 0.000 & 0.000 & 0.005 & 0.001 & 0.001 & 0.001 & 0.000 & 0.001 & 0.000 & 0.004 & 0.000 & 0.000 & 0.001 & 0.000 & 0.000 & 0.000 & 0.000 & 0.000 & 0.000 \\
\hline $\mathrm{K}$ & .000 & 000 & 0.000 & 0.000 & 0.002 & 0.002 & 0.001 & 0.001 & 0.000 & 0.000 & 0.000 & 0.001 & 0.001 & 0.000 & 0.000 & 0.000 & 0.000 & 0.0 & 0.000 & 0.000 & 0.000 \\
\hline $\mathrm{Cr}$ & 0.003 & 0.006 & 0.008 & 0.006 & 0.013 & 0.014 & 0.009 & 0.014 & 0.012 & 0.011 & 0.010 & 0.007 & 0.010 & 0.012 & 0.010 & 0.002 & 0.008 & 0.002 & 0.004 & 0.001 & 0.004 \\
\hline $\mathrm{Ni}$ & 0.007 & 0.012 & 0.016 & 0.003 & 0.021 & 0.024 & 0.015 & 0.008 & 0.009 & 0.013 & 0.010 & 0.005 & 0.009 & 0.011 & 0.010 & 0.009 & 0.007 & 0.018 & 0.009 & 0.012 & 0.018 \\
\hline Total & 6.362 & 6.374 & 6.368 & 6.361 & 6.397 & 6.401 & 6.397 & 6.366 & 6.365 & 6.383 & 6.363 & 6.366 & 6.364 & 6.346 & 6.340 & 6.376 & 6.384 & 6.348 & 6.352 & 6.317 & 6.364 \\
\hline
\end{tabular}




\section{Maurice, A. E.}

Table 3. Cont.

\begin{tabular}{|c|c|c|c|c|c|c|c|c|c|c|c|c|c|c|c|c|c|c|c|}
\hline \multicolumn{20}{|c|}{ Foliated serpentinite } \\
\hline No. & 43 & 44 & 45 & 46 & 47 & 48 & 49 & 50 & 51 & 52 & 53 & 54 & 55 & 56 & 57 & 58 & 59 & 60 & 61 \\
\hline $\mathrm{SiO}_{2}$ & 43.920 & 44.750 & 44.550 & 43.980 & 43.470 & 43.530 & 43.840 & 43.740 & 43.570 & 43.300 & 43.500 & 43.750 & 43.590 & 43.340 & 43.530 & 44.180 & 43.980 & 44.010 & 43.100 \\
\hline $\mathrm{TiO}_{2}$ & 0.000 & 0.000 & 0.002 & 0.000 & 0.000 & 0.000 & 0.000 & 0.005 & 0.000 & 0.003 & 0.000 & 0.000 & 0.000 & 0.001 & 0.000 & 0.000 & 0.000 & 0.000 & 0.000 \\
\hline $\mathrm{Al}_{2} \mathrm{O}_{3}$ & 0.505 & 0.421 & 0.216 & 0.277 & 0.668 & 0.693 & 0.756 & 0.675 & 0.480 & 0.622 & 0.570 & 0.804 & 0.634 & 0.977 & 0.667 & 0.409 & 0.605 & 0.400 & 1.172 \\
\hline $\mathrm{FeO}$ & 2.690 & 2.720 & 2.620 & 2.820 & 2.930 & 2.760 & 3.220 & 3.100 & 2.810 & 3.370 & 2.970 & 3.010 & 3.360 & 2.860 & 3.000 & 2.490 & 2.790 & 2.510 & 2.970 \\
\hline $\mathrm{MnO}$ & 0.047 & 0.050 & 0.053 & 0.022 & 0.015 & 0.042 & 0.010 & 0.074 & 0.010 & 0.037 & 0.022 & 0.025 & 0.040 & 0.035 & 0.030 & 0.032 & 0.046 & 0.015 & 0.041 \\
\hline $\mathrm{MgO}$ & 39.590 & 38.950 & 37.220 & 39.910 & 40.080 & 40.070 & 38.910 & 38.670 & 39.670 & 39.510 & 39.530 & 39.310 & 39.260 & 40.170 & 39.650 & 40.500 & 39.570 & 40.400 & 39.810 \\
\hline $\mathrm{CaO}$ & 0.016 & 0.028 & 0.003 & 0.015 & 0.013 & 0.021 & 0.040 & 0.031 & 0.015 & 0.012 & 0.015 & 0.012 & 0.010 & 0.018 & 0.010 & 0.011 & 0.014 & 0.011 & 0.007 \\
\hline $\mathrm{Na}_{2} \mathrm{O}$ & 0.000 & 0.000 & 0.000 & 0.000 & 0.000 & 0.006 & 0.000 & 0.000 & 0.005 & 0.008 & 0.000 & 0.000 & 0.004 & 0.000 & 0.000 & 0.000 & 0.000 & 0.002 & 0.011 \\
\hline $\mathrm{K}_{2} \mathrm{O}$ & 0.006 & 0.000 & 0.003 & 0.006 & 0.001 & 0.001 & 0.002 & 0.007 & 0.001 & 0.002 & 0.000 & 0.000 & 0.001 & 0.000 & 0.000 & 0.000 & 0.001 & 0.003 & 0.000 \\
\hline $\mathrm{Cr}_{2} \mathrm{O}_{3}$ & 0.088 & 0.053 & 0.017 & 0.003 & 0.075 & 0.037 & 0.076 & 0.063 & 0.040 & 0.160 & 0.195 & 0.164 & 0.200 & 0.363 & 0.261 & 0.005 & 0.157 & 0.019 & 0.145 \\
\hline $\mathrm{NiO}$ & 0.335 & 0.272 & 0.210 & 0.163 & 0.132 & 0.139 & 0.739 & 0.907 & 0.242 & 0.418 & 0.243 & 0.256 & 0.305 & 0.201 & 0.269 & 0.190 & 0.247 & 0.128 & 0.257 \\
\hline Total & 87.20 & 87.24 & 84.89 & 87.20 & 87.38 & 87.30 & 87.59 & 87.27 & 86.84 & 87.44 & 87.05 & 87.33 & 87.40 & 87.97 & 87.42 & 87.82 & 87.41 & 87.50 & 87.51 \\
\hline \multicolumn{20}{|c|}{ Formula based on 9 oxygens } \\
\hline Si & 2.628 & 2.668 & 2.720 & 2.630 & 2.599 & 2.602 & 2.623 & 2.628 & 2.619 & 2.598 & 2.612 & 2.617 & 2.612 & 2.578 & 2.605 & 2.620 & 2.625 & 2.620 & 2.577 \\
\hline $\mathrm{Al}$ & 0.036 & 0.030 & 0.016 & 0.020 & 0.047 & 0.049 & 0.053 & 0.048 & 0.034 & 0.044 & 0.040 & 0.057 & 0.045 & 0.069 & 0.047 & 0.029 & 0.043 & 0.028 & 0.083 \\
\hline $\mathrm{Ti}$ & 0.000 & 0.000 & 0.000 & 0.000 & 0.000 & 0.000 & 0.000 & 0.000 & 0.000 & 0.000 & 0.000 & 0.000 & 0.000 & 0.000 & 0.000 & 0.000 & 0.000 & 0.000 & 0.000 \\
\hline $\mathrm{Fe}$ & 0.135 & 0.136 & 0.134 & 0.141 & 0.146 & 0.138 & 0.161 & 0.156 & 0.141 & 0.169 & 0.149 & 0.151 & 0.168 & 0.142 & 0.150 & 0.124 & 0.139 & 0.125 & 0.149 \\
\hline $\mathrm{Mn}$ & 0.002 & 0.003 & 0.003 & 0.001 & 0.001 & 0.002 & 0.001 & 0.004 & 0.000 & 0.002 & 0.001 & 0.001 & 0.002 & 0.002 & 0.002 & 0.002 & 0.002 & 0.001 & 0.002 \\
\hline $\mathrm{Mg}$ & 3.531 & 3.462 & 3.388 & 3.558 & 3.572 & 3.571 & 3.470 & 3.463 & 3.554 & 3.534 & 3.538 & 3.505 & 3.508 & 3.561 & 3.537 & 3.581 & 3.521 & 3.585 & 3.548 \\
\hline $\mathrm{Ca}$ & 0.001 & 0.002 & 0.000 & 0.001 & 0.001 & 0.001 & 0.003 & 0.002 & 0.001 & 0.001 & 0.001 & 0.001 & 0.001 & 0.001 & 0.001 & 0.001 & 0.001 & 0.001 & 0.000 \\
\hline $\mathrm{Na}$ & 0.000 & 0.000 & 0.000 & 0.000 & 0.000 & 0.001 & 0.000 & 0.000 & 0.001 & 0.001 & 0.000 & 0.000 & 0.000 & 0.000 & 0.000 & 0.000 & 0.000 & 0.000 & 0.001 \\
\hline $\mathrm{K}$ & 0.000 & 0.000 & 0.000 & 0.000 & 0.000 & 0.000 & 0.000 & 0.001 & 0.000 & 0.000 & 0.000 & 0.000 & 0.000 & 0.000 & 0.000 & 0.000 & 0.000 & 0.000 & 0.000 \\
\hline $\mathrm{Cr}$ & 0.004 & 0.002 & 0.001 & 0.000 & 0.004 & 0.002 & 0.004 & 0.003 & 0.002 & 0.008 & 0.009 & 0.008 & 0.009 & 0.017 & 0.012 & 0.000 & 0.007 & 0.001 & 0.007 \\
\hline $\mathrm{Ni}$ & 0.016 & 0.013 & 0.010 & 0.008 & 0.006 & 0.007 & 0.036 & 0.044 & 0.012 & 0.020 & 0.012 & 0.012 & 0.015 & 0.010 & 0.013 & 0.009 & 0.012 & 0.006 & 0.012 \\
\hline Total & 6.353 & 6.316 & 6.272 & 6.360 & 6.376 & 6.373 & 6.349 & 6.347 & 6.364 & 6.377 & 6.363 & 6.351 & 6.361 & 6.380 & 6.366 & 6.365 & 6.350 & 6.366 & 6.379 \\
\hline
\end{tabular}


Egyptian Journal of Geology, v. 63, 2019, p.191-213

Table 4. Composition of magnesite of Um Esh serpentinites, Eastern Desert, Egypt.

\begin{tabular}{|c|c|c|c|c|c|c|c|c|c|c|c|c|c|c|c|c|c|c|}
\hline \multirow[b]{2}{*}{ No. } & \multicolumn{3}{|c|}{ Massive serpentinite } & \multirow[b]{2}{*}{4} & \multirow[b]{2}{*}{5} & \multirow[b]{2}{*}{6} & \multirow[b]{2}{*}{1} & \multirow[b]{2}{*}{8} & \multirow[b]{2}{*}{5} & \multirow[b]{2}{*}{10} & \multirow[b]{2}{*}{11} & \multirow[b]{2}{*}{12} & \multirow[b]{2}{*}{13} & \multirow[b]{2}{*}{14} & \multirow[b]{2}{*}{15} & \multicolumn{3}{|c|}{ Foliated serpentinite } \\
\hline & 1 & 2 & 3 & & & & & & & & & & & & & 16 & 17 & 18 \\
\hline $\mathrm{SiO}_{2}$ & 0.000 & 0.002 & 0.020 & 0.023 & 0.059 & 0.037 & 0.023 & 0.040 & 0.031 & 0.035 & 0.065 & 0.190 & 0.057 & 0.019 & 0.033 & 0.000 & 0.000 & 0.000 \\
\hline $\mathrm{TiO}_{2}$ & 0.002 & 0.004 & 0.014 & 0.001 & 0.000 & 0.021 & 0.000 & 0.018 & 0.001 & 0.000 & 0.003 & 0.000 & 0.000 & 0.007 & 0.001 & 0.007 & 0.010 & 0.008 \\
\hline $\mathrm{Al}_{2} \mathrm{O}_{3}$ & 0.000 & 0.000 & 0.000 & 0.000 & 0.000 & 0.000 & 0.000 & 0.000 & 0.005 & 0.000 & 0.007 & 0.011 & 0.005 & 0.000 & 0.000 & 0.000 & 0.000 & 0.011 \\
\hline $\mathrm{FeO}$ & 1.890 & 0.893 & 1.890 & 1.840 & 1.860 & 1.870 & 1.900 & 1.850 & 1.870 & 1.930 & 1.890 & 1.960 & 1.950 & 1.910 & 1.880 & 1.440 & 1.380 & 1.380 \\
\hline $\mathrm{MnO}$ & 0.379 & 0.432 & 0.401 & 0.372 & 0.421 & 0.342 & 0.369 & 0.333 & 0.383 & 0.389 & 0.353 & 0.348 & 0.365 & 0.372 & 0.370 & 0.600 & 0.514 & 0.593 \\
\hline $\mathrm{MgO}$ & 47.320 & 48.100 & 47.550 & 47.380 & 47.390 & 46.600 & 47.480 & 47.460 & 46.980 & 47.440 & 47.320 & 47.440 & 47.300 & 47.710 & 47.330 & 47.350 & 47.350 & 47.150 \\
\hline $\mathrm{CaO}$ & 0.062 & 0.180 & 0.057 & 0.058 & 0.056 & 0.045 & 0.059 & 0.072 & 0.046 & 0.070 & 0.067 & 0.059 & 0.075 & 0.068 & 0.062 & 0.382 & 0.288 & 0.376 \\
\hline $\mathrm{Na}_{2} \mathrm{O}$ & 0.000 & 0.000 & 004 & 0.000 & 0.000 & 0.000 & 0.000 & 0.000 & 0.000 & 0.003 & 0.014 & 0.000 & 0.000 & 0.009 & 0.000 & 0.012 & 0.000 & 0.003 \\
\hline $\mathrm{K}_{2} \mathrm{O}$ & 0.000 & 0.000 & 003 & 0.000 & 0.000 & 0.000 & 0.000 & 0.003 & 0.004 & 0.000 & 0.000 & 0.000 & 0.000 & 0.001 & 0.004 & 0.001 & 0.000 & 0.002 \\
\hline $\mathrm{NiO}$ & 0.031 & 0.015 & 0.029 & 0.031 & 0.044 & 0.036 & 0.043 & 0.033 & 0.033 & 0.033 & 0.030 & 0.047 & 0.034 & 0.018 & 0.048 & 0.019 & 0.038 & 0.009 \\
\hline Total & 49.68 & 49.64 & 49.99 & 49.71 & 49.83 & 48.96 & 49.87 & 49.81 & 49.35 & 49.90 & 49.76 & 50.06 & 49.79 & 50.11 & 49.73 & 49.81 & 49.58 & 49.53 \\
\hline
\end{tabular}

Table 4. Cont.

\begin{tabular}{|c|c|c|c|c|c|c|c|c|c|c|c|c|c|c|c|c|c|}
\hline & \multicolumn{17}{|c|}{ Foliated serpentinite } \\
\hline No. & 19 & 20 & 21 & 22 & 23 & 24 & 25 & 26 & 27 & 28 & 29 & 30 & 31 & 32 & 33 & 34 & 35 \\
\hline $\mathrm{SiO}_{2}$ & 0.000 & 0.000 & 0.000 & 0.006 & 0.000 & 0.001 & 0.000 & 0.000 & 0.000 & 0.000 & 0.004 & 0.030 & 0.000 & 0.000 & 0.001 & 0.000 & 0.000 \\
\hline $\mathrm{TiO}_{2}$ & 0.000 & 0.005 & 0.000 & 0.002 & 0.000 & 0.005 & 0.000 & 0.001 & 0.009 & 0.000 & 0.000 & 0.000 & 0.008 & 0.010 & 0.004 & 0.002 & 0.001 \\
\hline $\mathrm{Al}_{2} \mathrm{O}_{3}$ & 0.000 & 0.000 & 0.000 & 0.000 & 0.000 & 0.000 & 0.000 & 0.000 & 0.000 & 0.000 & 0.000 & 0.002 & 0.013 & 0.003 & 0.000 & 0.015 & 0.012 \\
\hline $\mathrm{FeO}$ & 1.490 & 1.520 & 1.480 & 1.390 & 1.450 & 1.310 & 1.300 & 1.420 & 1.450 & 1.360 & 1.460 & 1.410 & 1.360 & 1.272 & 1.262 & 1.330 & 1.286 \\
\hline $\mathrm{MnO}$ & 0.628 & 0.662 & 0.585 & 0.537 & 0.570 & 0.512 & 0.510 & 0.603 & 0.601 & 0.544 & 0.655 & 0.580 & 0.588 & 0.559 & 0.558 & 0.581 & 0.550 \\
\hline $\mathrm{MgO}$ & 46.900 & 46.360 & 46.610 & 47.250 & 47.340 & 47.410 & 47.410 & 47.110 & 47.150 & 47.290 & 47.000 & 47.340 & 47.340 & 46.780 & 47.390 & 46.750 & 47.280 \\
\hline $\mathrm{CaO}$ & 0.804 & 0.860 & 0.684 & 0.347 & 0.321 & 0.243 & 0.230 & 0.440 & 0.462 & 0.317 & 0.574 & 0.396 & 0.369 & 0.325 & 0.363 & 0.346 & 0.256 \\
\hline $\mathrm{Na}_{2} \mathrm{O}$ & 0.004 & 0.002 & 0.015 & 0.000 & 0.000 & 0.004 & 0.009 & 0.000 & 0.000 & 0.000 & 0.000 & 0.004 & 0.000 & 0.000 & 0.000 & 0.000 & 0.000 \\
\hline $\mathrm{K}_{2} \mathrm{O}$ & 0.000 & 0.006 & 0.002 & 0.000 & 0.004 & 0.004 & 0.006 & 0.000 & 0.001 & 0.000 & 0.000 & 0.000 & 0.000 & 0.000 & 0.000 & 0.002 & 0.000 \\
\hline $\mathrm{NiO}$ & 0.019 & 0.015 & 0.000 & 0.000 & 0.015 & 0.031 & 0.013 & 0.010 & 0.006 & 0.010 & 0.010 & 0.018 & 0.019 & 0.025 & 0.000 & 0.009 & 0.010 \\
\hline Total & 49.85 & 49.43 & 49.39 & 49.53 & 49.70 & 49.52 & 49.48 & 49.59 & 49.68 & 49.52 & 49.71 & 49.78 & 49.70 & 48.97 & 49.58 & 49.03 & 49.40 \\
\hline
\end{tabular}


Maurice, A. E.

Table 5: Major and trace elements (XRF) of Um Esh serpentinites, Eastern Desert, Egypt.

\begin{tabular}{|c|c|c|c|c|c|c|c|c|}
\hline & \multicolumn{6}{|c|}{ Massive serpentinite } & \multicolumn{2}{|c|}{ Foliated serpentinite } \\
\hline Sample & UE1 & UE2 & UE3 & UE4 & UE5 & UE6 & UE7 & UE8 \\
\hline $\mathrm{SiO}_{2}$ & 40.441 & 40.504 & 37.145 & 37.477 & 35.310 & 33.250 & 36.739 & 38.194 \\
\hline $\mathrm{TiO}_{2}$ & 0.005 & 0.004 & 0.006 & 0.007 & 0.009 & 0.007 & 0.004 & 0.004 \\
\hline $\mathrm{Al}_{2} \mathrm{O}_{3}$ & 0.600 & 0.609 & 0.390 & 0.385 & 0.459 & 0.369 & 0.568 & 0.596 \\
\hline $\mathrm{Fe}_{2} \mathrm{O}_{3}$ & 7.375 & 7.403 & 6.986 & 6.897 & 6.630 & 7.627 & 7.554 & 6.930 \\
\hline $\mathrm{FeO}$ & 0.000 & 0.000 & 0.000 & 0.000 & 0.000 & 0.000 & 0.000 & 0.000 \\
\hline $\mathrm{MnO}$ & 0.062 & 0.059 & 0.086 & 0.083 & 0.124 & 0.142 & 0.098 & 0.089 \\
\hline $\mathrm{MgO}$ & 38.835 & 38.842 & 39.244 & 39.223 & 37.629 & 37.057 & 38.574 & 38.759 \\
\hline $\mathrm{CaO}$ & 0.000 & 0.000 & 0.000 & 0.000 & 2.834 & 3.324 & 0.332 & 0.112 \\
\hline $\mathrm{Na}_{2} \mathrm{O}$ & 0.001 & 0.004 & 0.008 & 0.000 & 0.026 & 0.020 & 0.011 & 0.004 \\
\hline $\mathrm{K}_{2} \mathrm{O}$ & 0.013 & 0.012 & 0.013 & 0.014 & 0.018 & 0.015 & 0.012 & 0.016 \\
\hline $\mathrm{P}_{2} \mathrm{O}_{5}$ & 0.004 & 0.004 & 0.003 & 0.003 & 0.004 & 0.003 & 0.003 & 0.003 \\
\hline $\mathrm{Cr}_{2} \mathrm{O}_{3}$ & 0.349 & 0.323 & 0.320 & 0.330 & 0.335 & 0.254 & 0.463 & 0.616 \\
\hline $\mathrm{NiO}$ & 0.280 & 0.281 & 0.330 & 0.321 & 0.239 & 0.212 & 0.258 & 0.282 \\
\hline LOI & 12.112 & 12.041 & 15.531 & 15.255 & 16.395 & 17.730 & 15.497 & 14.509 \\
\hline Total & 100.08 & 100.09 & 100.06 & 100.00 & 100.01 & 100.01 & 100.11 & 100.11 \\
\hline $\mathrm{Al}_{2} \mathrm{O}_{3} / \mathrm{SiO}_{2}$ & 0.015 & 0.015 & 0.011 & 0.010 & 0.013 & 0.011 & 0.015 & 0.016 \\
\hline $\mathrm{MgO} / \mathrm{SiO}_{2}$ & 0.960 & 0.959 & 1.056 & 1.047 & 1.066 & 1.115 & 1.050 & 1.015 \\
\hline $\mathrm{Ba}$ & 11.30 & 10.90 & 0.00 & 4.90 & 2.70 & 0.00 & 6.50 & 19.80 \\
\hline $\mathrm{Sr}$ & 2.80 & 0.00 & 0.00 & 0.00 & 48.00 & 59.10 & 6.10 & 2.10 \\
\hline $\mathrm{Zr}$ & 2.40 & 7.50 & 6.70 & 7.80 & 7.30 & 7.20 & 6.50 & 7.60 \\
\hline $\mathrm{Hf}$ & 1.30 & 1.70 & 0.90 & 1.10 & 1.80 & 1.90 & 1.40 & 0.80 \\
\hline $\mathrm{Y}$ & 0.00 & 4.50 & 3.80 & 3.90 & 2.80 & 3.60 & 3.70 & 3.90 \\
\hline $\mathrm{Zn}$ & 34.90 & 28.90 & 41.10 & 44.80 & 62.70 & 43.80 & 54.00 & 72.70 \\
\hline $\mathrm{Cu}$ & 8.80 & 6.30 & 6.30 & 11.00 & 9.00 & 13.20 & 7.50 & 6.00 \\
\hline $\mathrm{Ni}$ & 2506 & 2510 & 3072 & 2981 & 2243 & 2021 & 2398 & 2588 \\
\hline Co & 105.5 & 104.1 & 128.0 & 128.0 & 89.2 & 92.5 & 109.4 & 111.2 \\
\hline $\mathrm{Cr}$ & 2717 & 2516 & 2591 & 2666 & 2744 & 2109 & 3751 & 4927 \\
\hline $\mathrm{V}$ & 26.3 & 25.0 & 26.7 & 25.4 & 30.3 & 22.2 & 32.6 & 33.7 \\
\hline Sc & 6.40 & 5.90 & 6.20 & 8.70 & 9.30 & 4.30 & 3.60 & 7.40 \\
\hline Th & 2.10 & 0.00 & 1.40 & 0.00 & 0.00 & 0.00 & 0.00 & 0.10 \\
\hline $\mathrm{U}$ & 0.00 & 3.30 & 3.60 & 5.30 & 5.40 & 6.10 & 4.50 & 4.20 \\
\hline
\end{tabular}

\section{DISCUSSION}

\section{Tectonic setting}

The tectonic setting of the ophiolites of the Eastern Desert of Egypt has been controversial. Both subduction-related (e.g. Azer and Stern, 2007; Abd El-Rahman et al., 2009; Basta et al., 2011; AbdelKarim et al., 2016; Khedr and Arai, 2017) and subduction-unrelated (e.g. Zimmer et al., 1995; Khalil, 2007) settings have been proposed for the Neoproterozoic ophiolites of Egypt. The $\mathrm{Al}_{2} \mathrm{O}_{3} / \mathrm{SiO}_{2}(0.010$ 0.016) and $\mathrm{MgO} / \mathrm{SiO}_{2}$ (0.96-1.11) values of the Um Esh serpentinites are comparable to those of forearc peridotites (Fig. 7), where the fore-arc peridotites are characterized by low $\mathrm{Al}_{2} \mathrm{O}_{3} / \mathrm{SiO}_{2}$ values. In the extensively serpentinized peridotites, the composition of fresh Cr-spinel has been used as an important petrogenetic indicator (e.g. Barnes and Roeder, 2001). Kamenetsky et al. (2001) suggested that the $\mathrm{TiO}_{2}$ contents of Cr-spinels can be used to discriminate between peridotites from superasubduction zones (SSZ) and mid-oceanic ridges (MOR). The $\mathrm{Al}_{2} \mathrm{O}_{3}$ and $\mathrm{TiO}_{2}$ abundances of the analyzed fresh Cr-spinels overlap those of Cr-spinels of SSZ and MOR peridotites (Fig. 8A). According to Dick and Bullen (1984), the Cr\# of fresh Cr-spinel (0.55-0.66) is spanning the range of MOR (Cr\# < 0.60) and SSZ (Cr\# > 0.60) ophiolites. However, the Mg\# (0.39-0.59) and Cr\# of the primary Cr-spinel are similar to those of modern SSZ fore-arc peridotites (Fig. 8B). Thus, the protoliths of Um Esh serpentinites are akin to SSZ rather than MOR peridotites.

\section{Nature of Neoproterozoic SSZ Mantle}

The low $\mathrm{Al}_{2} \mathrm{O}_{3} / \mathrm{SiO}_{2}$ (0.010-0.016) values of the Um Esh serpentinites suggest that they represent residual mantle which has experienced high degree of partial melting. The fresh Cr-spinel of Um Esh serpentinite is characterized by very low $\mathrm{TiO}_{2}$ contents $(<0.025 \mathrm{wt} \%)$, confirming the depleted nature of these mantle rocks (Jan and Windley, 1990). The primary spinel of harzburgite has $\mathrm{Cr} \#(\sim 0.50)$ lower than that ( 0.70) of Cr-spinel of dunite (Arai and Yurimoto, 1994). Moreover, the Cr\# of spinel 


\section{Petrology and geochemistry of Um Esh ophiolite serpentinites}

is proportionally related to the degree of mantle partial melting (Dick and Bullen, 1984). The normative proportions of olivine, orthopyroxene and clinopyroxene of the analyzed samples suggest that the Um Esh serpentinites were formed after harzburgite protolith (Fig. 6). Thus, the higher Cr\# (0.55-0.66) of the Um Esh serpentinized harzburgite compared with that of spinel of harzburgite ( $\sim 0.50$, Arai and Yurimoto, 1994) reflects higher degree of partial melting of residual mantle or highly depleted mantle. Moreover, the primary Cr-spinels plot mostly in the field of highly depleted mantle peridotites on the $\mathrm{TiO}_{2}$ versus Cr\# diagram (Fig. 9). In short, the geochemical characteristics of Um Esh serpentinites and their accessory Cr-spinels are consistent with depleted to highly depleted nature of harzburgite mantle.
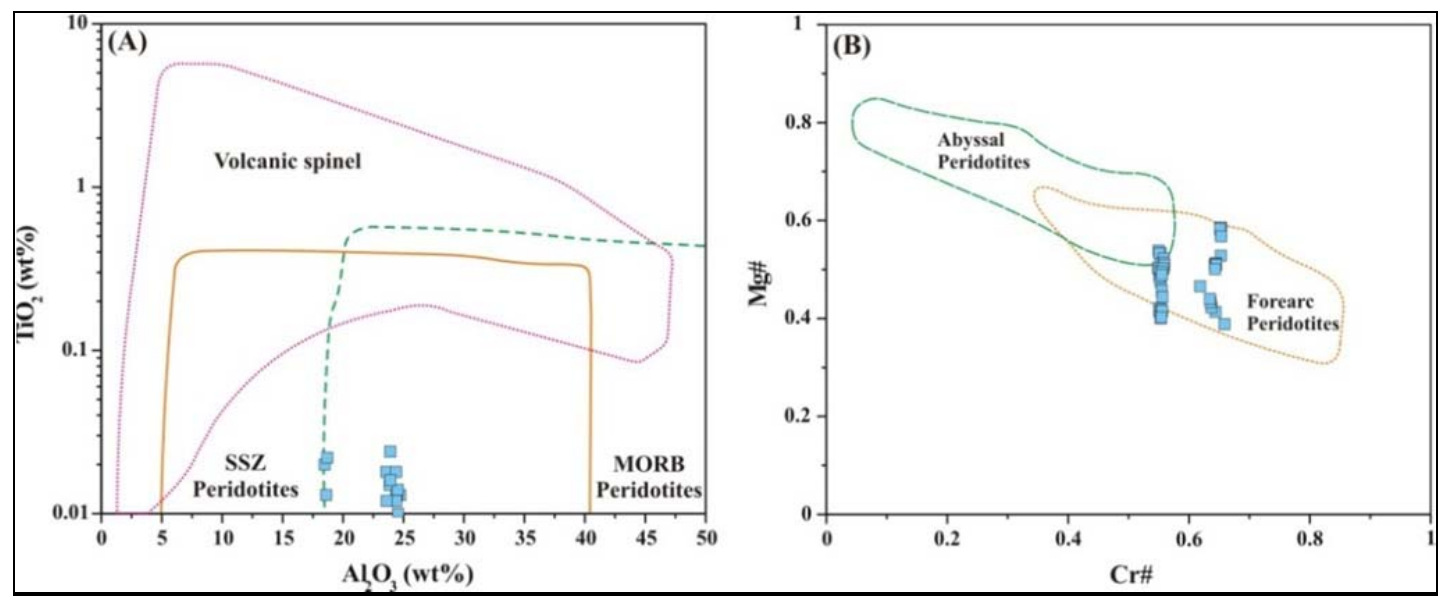

Fig. 8: Tectonic setting of the Um Esh serpentinites. (A) $\mathrm{TiO}_{2}$ versus $\mathrm{Al}_{2} \mathrm{O}_{3}$ discrimination diagram (after Kamenetsky et al., 2001), (B) Mg\# versus Cr\# of the primary Cr-spinel (after Metcalf and Shervais, 2008).

Fig. 9. $\mathrm{TiO}_{2}$ versus $\mathrm{Cr} \#$ diagram of the primary Cr-spinel of Um Esh serpentinites (fields after Dick and Bullen, 1984, Jan and Windley, 1990, Arai, 1992).

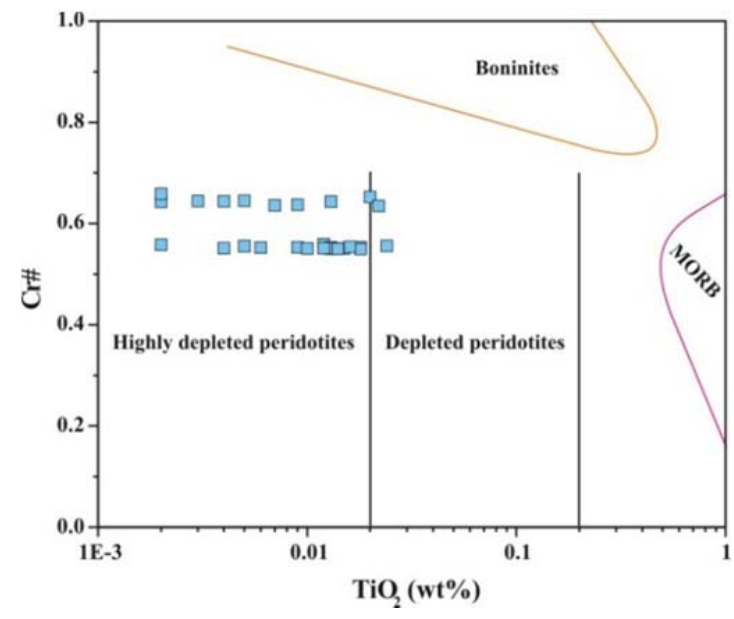

\section{Metamorphism}

During low temperature $\left(<300{ }^{\circ} \mathrm{C}\right)$ serpentinization of peridotites, olivine and pyroxene are replaced with lizardite/chrysotile. Prograde metamorphism of serpentinites involves transformation of lizardite/chrysotile to antigorite (Derbyshire et al., 2013). Evans et al. (2013) proposed that antigorite grows at the expense of lizardite and chrysotile at temperature above $320{ }^{\circ} \mathrm{C}$. The serpentines of Um Esh serpentinite are essentially antigorites (Fig. 5), implying serpentine recrystallization during prograde metamorphism. The Cr-spinel grains of Um Esh serpentinites are usually zoned, with ferritchromite and chromian magnetite (Fig. $2 \mathrm{C}$ and D). The origin of Cr-spinel zonation has been attributed either to serpentinization of ultramafic rocks (e.g. Takla, 1982) or to prograde metamorphism of serpentinized peridotites (e.g. Farahat, 2008; Anzil et al., 2012; Derbyshire et al., 2013). The metamorphic spinel rims are not likely produced during low temperature strongly reducing conditions associated with serpentinization (Derbyshire et al., 2013), which are attributed to production of dihydrogen $\left(\mathrm{H}_{2}\right)$ gas as a result of oxidation of ferrous iron in olivine and pyroxene into ferric iron and precipitation of magnetite (Seyfried et al., 2007; Evans, 2010; McCollom et al., 2016; Huang et al., 2017). Moreover, the minimum temperature of formation of ferritchromite is $\sim 500{ }^{\circ} \mathrm{C}$ (Kimball, 1990; Mellini et al., 2005). Profiles along zoned Cr-spinel grains revealed an increase of $\mathrm{Fe}^{3+}$ from fresh spinel through ferritchromite to chromian magnetite (Fig. 4D), suggesting oxidizing 
conditions during the formation of metamorphic spinel (ferritchromite and chromian magnetite). Plotting the Cr-spinel analyses on the $\mathrm{Cr}-\mathrm{Al}-\mathrm{Fe}^{3+}$ diagram revealed that the ferritchromites were formed at lower amphibolite facies (Fig. 10). Thus, the ferritchromite rims of Cr-spinel of Um Esh serpentinites were produced under oxidizing conditions during prograde (lower amphibolite facies) metamorphism of serpentinites, implying temperature of metamorphism between $500-600{ }^{\circ} \mathrm{C}$ (Khalil, 2007; Farahat, 2008; Gonzálzez-Jiménez et al., 2009; Abu El-Ela and Farahat, 2011).

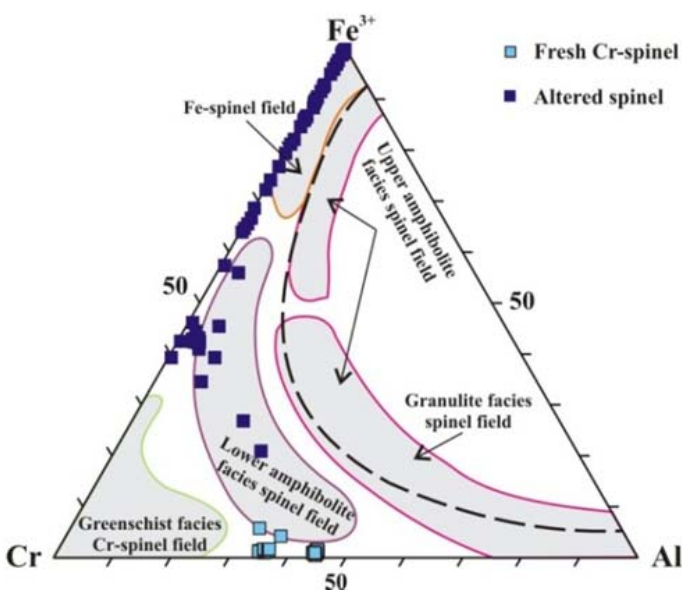

Fig. 10: The composition of primary Crspinels and their alteration products plotted on $\mathrm{Cr}-\mathrm{Al}-\mathrm{Fe}^{3+}$ ternary diagram. The fields of different metamorphic facies for Cr-spinel phases and solvus curve (dashed line) are reproduced from Purvis et al. (1972), Evans and Frost (1975), Suita and Strieder (1996) and Barnes and Roeder (2001).

\section{Element mobility during Cr-spinel alteration and metamorphism}

Alteration and metamorphism of Cr-spinel result in development of zoned grains (Evans and Frost, 1975) and are accompanied with variation of the abundances of the different elements in the different produced zones (e.g. Khudeir et al., 1992; Azer and Stern, 2007; Khalil, 2007; Farahat, 2008; Abu ElEla and Farahat, 2011; Derbyshire et al., 2013). Composition profiles along the different zones of the accessory Cr-spinels of Um Esh serpentinites revealed that $\mathrm{Al}, \mathrm{Mg}, \mathrm{Cr}$ and $\mathrm{Fe}$ have been exchanged with the surrounding silicates, where $\mathrm{Al}, \mathrm{Mg}$ and $\mathrm{Cr}$ were removed while $\mathrm{Fe}$ was added to $\mathrm{Cr}$-spinel (Fig. 4A-C). The higher $\mathrm{MnO}$ content of the ferritchromite zones relative to the fresh cores is probably due to addition of $\mathrm{Mn}$ released from olivine alteration, whereas the depletion of chromian magnetite rims in $\mathrm{MnO}$ relative to ferritchromite (Fig. 4E) can be attributed to accommodation of $\mathrm{Mn}$ in the structure of magnesite during progressive metamorphism. This interpretation is supported by the fact that magnesites of Um Esh serpentinites contain up to $0.66 \mathrm{wt} \% \mathrm{MnO}$. Similarly, the higher $\mathrm{NiO}$ content of the ferritchromite zones and the inner rims of the chromian magnetite zones (Fig. 4F), can be interpreted by addition of $\mathrm{Ni}$ from altered olivine, while the depletion of $\mathrm{NiO}$ in the outer rims of the chromian magnetite zones can be attributed to donation of $\mathrm{Ni}$ to the surrounding serpentines which contain up to $0.91 \mathrm{wt} \% \mathrm{NiO}$. The depletion of $\mathrm{Ni}$ in only the outer rims of the chromian magnetite zones, compared with $\mathrm{MnO}$ depletion in the whole chromian magnetite zone, implies slower diffusion rate of $\mathrm{Ni}$ from spinel to the surrounding minerals during metamorphism.

\section{CONCLUSIONS}

1- The low $\mathrm{Al}_{2} \mathrm{O}_{3} / \mathrm{SiO}_{2}(0.010-0.016)$ values of serpentinite samples and the very low $\mathrm{TiO}_{2}$ contents $(<0.025 \mathrm{wt} \%)$ of primary Cr-spinels together with $\mathrm{Cr} \#$ ranging from 0.55 to 0.66 indicate that the Um Esh ophiolite serpentinites represent Neoproterozoic residual harzburgite mantle which experienced high degree of partial melting.

2- The depleted to highly depleted nature of Um Esh serpentinites as reflected by whole-rock geochemical characteristics and mineral chemistry of primary Cr-spinels suggest that these serpentinites are similar to modern SSZ fore-arc peridotites rather than MOR peridotites.

3- The dominance of antigorite and the presence of metamorphic spinel rims suggest that Um Esh serpentinites have experienced prograde metamorphism up to lower amphibolite facies under oxidizing conditions.

4- Composition profiles along the different zones of the accessory Cr-spinels of Um Esh serpentinites revealed that $\mathrm{Al}, \mathrm{Mg}, \mathrm{Cr}$ and $\mathrm{Fe}$ have been exchanged with the surrounding silicates, where $\mathrm{Al}, \mathrm{Mg}$ and $\mathrm{Cr}$ were removed while $\mathrm{Fe}$ was added to $\mathrm{Cr}$-spinel. $\mathrm{Mn}$ and $\mathrm{Ni}$ from altered olivine have been added to the Cr-spinel then donated from the chromian magnetite zones to the surrounding 


\section{Petrology and geochemistry of Um Esh ophiolite serpentinites}

magnesite and serpentines, respectively. The diffusion rate of Ni from the chromian magnetite rims to the surrounding silicates was slower than that of Mn.

\section{ACKNOWLEDGEMENTS}

Prof. Dr. Chris Heinrich, ETH-Zurich, Switzerland and Prof. Dr. Bottros R. Bakhit, Beni-Suef University, are deeply acknowledged for the laboratory facilities and geochemical analyses without which the present work was not possible.

\section{REFERENCES}

Abdel Khalek, M. I., Abdel Maksoud, M. A., Abdel Tawab, M. A. and El-Bedawi, M.A. (1994): An ophiolite-mélange complex south of Dahab, Sinai, Egypt. Ann. Geol. Surv. Egypt, 20, 1-18.

Abd El-Rahman, Y., Ploat, A., Dilek, Y., Fryer, B., El-Sharkawy, M. and Sakran, S. (2009): Geochemistry and tectonic evolution of the Neoproterozoic Wadi Ghadir Ophiolite, Eastern Desert, Egypt. Lithos, 113 (1-2), 158-178.

Abdel-Karim, A. M., Ali, S., Helmy, H. M. and El-Shafei, S. A. (2016): Fore-arc setting of the Gerf ophiolite, Eastern Desert, Egypt: evidence from mineral chemistry and geochemistry of ultramafites. Lithos, 263, 52-65.

Abu El-Ela, F. F. and Farahat, E. S. (2011): Neoproterozoic podiform chromitites in serpentinites of the Abu Meriewa-Hagar Dungash district, Eastern Desert, Egypt: geotectonic implications and metamorphism. Island Arc, 19, 151-164.

Abu El-Enen M. M. and Makroum F. M. (2003): Tectonometamorphic evolution of the northeastern Kid Belt, Southeast Sinai, Egypt. Ann. Geol. Surv. Egypt, 26, 19-37.

Akaad, M.K. and Noweir, A.M. (1980): Geology and lithostratigraphy of the Arabian Desert orogenic belt of Egypt between latitudes $25^{\circ} 35^{\prime}$ and $26^{\circ} 30^{\prime}$ N. Inst. App. Geol. Bull., Jeddah, 2, 127-136.

Ahmed, A. H. (2013): Highly depleted harzburgite-dunite-chromitite complexes from the Neoproterozoic ophiolite, south Eastern Desert, Egypt: a possible recycled upper mantle lithosphere. Precamb. Res., 233, 173-192.

Ahmed, A. H., Arai, S. and Attia, A.K. (2001): Petrological characteristics of podiform chromitites and associated peridotites of the Pan African ophiolite complexes of Egypt. Mineral. Deposita, 36, 72-84.

Ahmed, A. H., Harbi, H. M. and Habtoor, A. M. (2012): Compositional variations and tectonic settings of podiform chromitites and associated ultramafic rocks of the Neoproterozoic ophiolite at Wadi Al Hwanet, northwestern Saudi Arabia. J. Asian Earth Sci., 56, 118-134.

Ali, K. A., Azer, M.K., Gahlan, H. A., Wilde, S. A., Samuel, M.D. and Stern, R.J. (2010): Age constraints on the formation and emplacement of Neoproterozoic ophiolites along the AllaqiHeiani suture, South Eastern Desert of Egypt. Gond. Res., 18, 583-595.

Andresen, A., El-Rus, M. A. A., Myhre, P. I. and Boghdady, G. Y. (2009): U-Pb TIMS age constraints on the evolution of the Neoproterozoic Meatiq Gneiss Dome, Eastern Desert, Egypt. Inter. J. Earth Sci., 98, 481-497.

Anzil, P. A., Guereschi, A. B. and Martino, R. D. (2012): Mineral chemistry and geothermometry using relict primary minerals in the La Cocha ultramafic body: a slice of the upper mantle in the Sierra Chica of Cordoba, Sierras Pampeanas, Argentina. J. South Amer. Earth Sci., 40, 38-52.

Arai, S. (1992): Chemistry of chromian spinel in volcanic rocks as a potential guide to magma chemistry. Mineral. Mag., 56, 173-184.

Arai, S. and Yurimoto, H. (1994): Podiform chromitites of Tari-misaka ultramafic complex, southwest Japan, as mantle-melt interaction products. Econ. Geol., 89, 1279-1288.

Azer, M. K. and Stern, R. J. (2007): Neoproterozoic (835-720 Ma) serpentinites in the Eastern Desert, Egypt: fragments of forearc mantle. J. Geol., 115, 457-472.

Bakhit, B.R. (1994): Gold mineralization in some occurrences of the Eastern Desert, Egypt. M. Sc Thesis, Cairo Univ., 214p.

Bakhit, B. R. (2002): Geology, mineralogy and genesis of some gold occurrences, Eastern Desert, Egypt. Ph. D Thesis, Cairo Univ., 300p.

Barnes, S. J. and Roeder, P. L. (2001): The range of spinel compositions in terrestrial mafic and ultramafic rocks. J. Petrol., 42, 2279-2302.

Basta, F.F. (1983): Geology and geochemistry of the ophiolitic mélange and other rock units in the area around and west of Gabal Ghadir, Eastern Desert, Egypt. Ph.D. thesis, Cairo Univ., 137p. 
Basta, F. F., Maurice, A. E., Bakhit, B. R., Ali, K. A. and Manton, W. I. (2011): Neoproterozoic Contaminated MORB of Wadi Ghadir Ophiolite, NE Africa: Geochemical and Nd and Sr isotopic Constraints. J. Afr. Earth Sci., 59, 227-242.

Basta, F. F., Maurice, A. E., Bakhit, B. R., Azer, M. K. and El-Sobky, A. F. (2017): Intrusive rocks of the Wadi Hamad area, North Eastern Desert, Egypt: change of magma composition with maturity of Neoproterozoic continental island arc and the role of collisional plutonism in the differentiation of arc crust. Lithos, 288-289, 248-263.

Coleman, R. G. (1977): Ophiolites. Springer-Verlag, Berlin, 229 p.

Derbyshire, E. J., O'Driscoll, B., Lenaz, D., Gertisser, R. and Kronz, A. (2013): Compositionally heterogeneous podiform chromitite in the Shetland Ophiolite Complex (Scotland): Implications for chromitite petrogenesis and late-stage alteration in the upper mantle portion of a supra-subduction zone ophiolite. Lithos, 162-163, 279-300.

Dick, H. B. and Bullen, T. (1984): Chromian spinel as a petrogenetic indicator in abyssal and Alpinetype peridotites and spatially associated lavas. Contrib. Mineral. Petrol., 86, 54-76.

Dilek, Y. and Furnes, H. (2011): Ophiolite genesis and global tectonics: Geochemical and tectonic fingerprinting of ancient oceanic lithosphere. Geol. Soc. Amer. Bull., 123, 387-411.

Dilek, Y. and Furnes, H. (2014): Ophiolites and their origins. Elements 10, 93-100.

Dungan, M. A. (1979): A microprobe study of antigorite and some serpentine pseudomorphs. Amer. Mineral., 17, 771-784.

El-Amawy, M. A., Wetait, M. A., Mehanna, A. M., Soliman, F. A. and Ghabour, Y. (2004): Geology and structural evolution of the basement rocks of Imlig area, southwest Sinai, Egypt. Ann. Geol. Surv. Egypt, 27, 19-33.

El-Sayed, M. M., Furnes, H. and Mohamed, F. H. (1999): Geochemical constraints on the tectonomagmatic evolution of the late Precambrian Fawakhir ophiolite, Central Eastern Desert, Egypt. J. Afr. Earth Sci., 29 (3), 515-533.

El-Sharkawy, M. A. and El-Bayoumi, R. (1979): The ophiolites of Wadi Ghadir area, Eastern Desert, Egypt. Ann. Geol. Surv. Egypt, 9, 125-135.

Evans, B. W. (2010): Lizardite versus antigorite serpentinite: magnetite, hydrogen and life (?). Geology, 38, 879-882.

Evans, B. W. and Frost, B. R. (1975): Chrome-spinel in progressive metamorphism - a preliminary analysis. Geochim. Cosmochim. Acta, 39, 959-972.

Evans, B. W., Hattori, K. and Baronnet, A. (2013): Serpentinite: what, why, where?. Elements, 9, 99106.

Farahat, E. S. (2008): Chrome-spinels in serpentinites and talc carbonates of the El Ideid-El-Sodmein District, central Eastern Desert, Egypt: their metamorphism and petrogenetic implications. Chem. Erde, 68, 193-205.

Franz, L. and Wirth, R. (2000): Spinel inclusions in olivine of peridotite xenoliths from TUBAF seamount (Bismarck Archipelago/Papua New Guinea): evidence for the thermal and tectonic evolution of the oceanic lithosphere. Contrib. Mineral. Petrol., 140, 283-295.

Gahlan, H. A., Arai, S., Abu El-Ela, F. F. and Tamura, A. (2012): Origin of wehrlite cumulates in the Moho transition zone of the Neoproterozoic Ras Salatit ophiolite, Central Eastern Desert, Egypt: crustal wehrlites with typical mantle characteristics. Contrib. Mineral. Petrol., 163, 25-241.

González-Jiménez, J. M., Kerestedjian, T., Proenza, J. A. and Gervilla, F., (2009): Metamorphism on chromite ores from the Dobromirtsi Ultramafic Massif, Rhodope Mountains (SE Bulgaria). Geol. Acta, 7, 413-429.

Harraz, H. (1995): Primary geochemical haloes, El Sid gold mine, Eastern Desert, Egypt. J. Afr. Earth Sci. 20, 61-71.

Hart, S. R. and Zindler, A. (1986): In search of a bulk-earth composition. Chem. Geol., 57, 247-267.

Huang, R., Lin, C.-T., Sun, W., Ding, X., Zhan, W. and Zhu, J. (2017): The production of iron oxide during peridotite serpentinization: influence of pyroxene. Geosci. Front., 8, 1311-1321.

Jan, M. Q. and Windley, B.F. (1990): Chromian spinel-silicate chemistry in ultramafic rocks of the Jijal complex, northwestern Pakistan. J. Petrol., 31, 667-715.

Jagoutz, E., Palme, H., Baddenhausen, H., Blum, K., Cendales, M., Dreibus, G., Spettel, B., Lorenz, V. and Vanke, H. (1979): The abundance of major, minor and trace elements in the earth's mantle as derived from primitive ultramafic nodules. Geochim. Cosmochim. Acta, 11 (2), 2031-2050. 
Khedr, M. Z. and Arai, S. (2017): Peridotite-chromitite complexes in the Eastern Desert of Egypt: Insight into Neoproterozoic sub-arc mantle processes. Gond. Res., 52, 59-79.

Kamenetsky, V., Crawford, A.J. and Meffre, S. (2001): Factors controlling chemistry of magmatic spinel: an empirical study of associated olivine, Cr-spinel and melt inclusions from primitive rocks. J. Petrol., 42, 655-671.

Khalil, K. I. (2007): Chromite mineralization in ultramafic rocks of the Wadi Ghadir area, Eastern Desert, Egypt: mineralogical, microchemical and genetic studies. N. Jb. Miner. Abh., 183, 283-296.

Kimball, K. (1990): Effects of hydrothermal alteration on the composition of chromian spinels. Contrib. Mineral. Petrol., 105, 337-346.

Kröner, A., Todt, W., Hussein, I. M., Mansour, M. and Rashwan, A. A. (1992): Dating of late Proterozoic ophiolites in Egypt and the Sudan using the single grain zircon evaporation technique. Precamb. Res., 59, 15-32.

Khudeir, A. A., El-Haddad, M. A. and Leake, B. E. (1992): Compositional variation in chromite from the Eastern Desert, Egypt. Mineral. Mag., 56, 567-574.

Madbouly, M. I. (2000): A comparative study on petrology and geochemistry of some maficultramafic intrusions of the Eastern Desert and Sinai, Egypt. Ph. D. Thesis, Cairo Univ., 262 p.

McCollom, T.M., Klein, F., Robbins, M., Moskowitz, B., Berquó, T.S., Jöns, N., Bach, W. and Templeton, A. (2016): Temperature trends for reaction rates, hydrogen generation, and partitioning of iron during experimental serpentinization of olivine. Geochim. Cosmochim. Acta, 181, 175-200.

Mehanna, A.M., Wetait, M.A., El-Amawy, M.A., Solimani, F.A. and Ghabour, Y. (2004): Petrogenesis and metamorphism of the basement rocks of Imlig area, southwest Sinai, Egypt. Ann. Geol. Surv. Egypt, 27, 35-59.

Mellini, M., Rumori, C. and Viti, C. (2005): Hydrothermally reset magmatic spinels in retrograde serpentinites: formation of 'ferritchromit' rims and chlorite aureoles. Contrib. Mineral. Petrol., 149, 266-275.

Metcalf, R. V. and Shervais, J. W. (2008): Suprasubduction-zone ophiolites: is there really an ophiolite conundrum? Geol. Soc. Amer. Special Paper 438, 191-222.

Niu, Y. (2004): Bulk-rock major and trace element compositions of abyssal peridotites: implications for mantle melting, melt extraction and post-melting processes beneath mid-ocean ridges. J. Petrol., 45, 2423-2458.

Parkinson, I. J. and Pearce, J. A. (1998): Peridotites of the Izu-Bonin-Mariana forearc (ODP Leg 125) evidence for mantle melting and melt-mantle interactions in a supra-subduction zone setting. J. Petrol., 39, 1577-1618.

Pearce, J. A. (2014): Immobile element fingerprinting of ophiolites. Elements, 10, 101-108.

Pearce, J. A., Barker, P. F., Edwards, S. J., Parkinson, I. J. and Leat, P. T. (2000): Geochemistry and tectonic significance of peridotites from the South Sandwich arc-basin system, South Atlantic. Contrib. Mineral. Petrol., 139, 36-53.

Purvis, A. C., Nesbitt, R. W. and Halberg, J. A. (1972): The geology of part of Carr Boyd Complex and its associated nickel mineralization, Western Australia. Econ. Geol., 67, 1093-1113.

Schwartz, S., Guillot, S., Reynard, B., Lafay, R., Debret, B., Nicollet, C., Lanari, P. and Auzende, A. L. (2013): Pressure-temperature estimates of the lizardite/antigorite transition in high pressure serpentinites. Lithos, 178, 197-210.

Seyfried Jr., W. E., Foustoukos, D.I. and Fu, Q. (2007): Redox evolution and mass transfer during serpentinization: an experimental and theoretical study at $200{ }^{\circ} \mathrm{C}, 500$ bar with implications for ultramafic-hosted hydrothermal systems at mid-ocean ridges. Geochim. Cosmochim. Acta, 71, 3872-3886.

Shimron, A. E. (1981): The Dabab mafic-ultramafic complex. A probable ophiolite of late Proterozoic (Pan-African) age. Ofioliti, 6, 161-164 .

Shimron, A.E. (1984): Evolution of the Kid Group, southeast Sinai Peninsula: thrusts, mélanges, and implications for accretionary tectonics during the Proterozoic of the Arabian-Nubian Shield. Geology, 12, 242-247.

Suita, M. T. and Strieder, A. J. (1996): Cr-spinels from Brazilian mafic-ultramafic complexes: metamorphic modifications. Inter. Geol. Rev., 38, 245-267.

Stern, R. J. (1994): Arc assembly and continental collision in the Neoproterozoic East-African Orogen - implications for the consolidation of Gondwanaland. Ann. Rev. Earth Planet. Sci., 22, 319-351.

Takla, M. A. (1982): Chromites from the Bergen arcs ultramafics, southern Norway. N. Jb. Miner. Abh., 144, 56-72. 
Maurice, A. E.

Takla, M. A., Basta, F. F., Madbouly, M. I. and Hussein, A. A. (2001): The mafic-ultramafic intrusions of Sinai, Egypt. Ann. Geol. Surv. Egypt, 24, 1-40.

Taman, Z. (1996): Geology and mineralization of Wadi Atalla area, Eastern Desert, Egypt. MSc thesis, Ain Shams Univ., 120 p.

Wassef, B., Kamel, O. A., Armanious, L. K. and Sabet, A. H. (1973): Report on the results of the prospecting work for gold in El Sid-Semna area in 1970/1971. Geol. Surv. Egypt, internal report no. 23/1973.

Zimmer, M., Kröner, A., Jochum, K. P., Reischmann, T. and Todt, W. (1995): The Gabal Gerf complex: A Precambrian N-MORB ophiolite in the Nubian Shield, NE Africa. Chem. Geol., 123, 29-51.

Zoheir, B., Deshesh, F., Broman, C., Pitcairn, I., El-Metwally, A. and Mashaal, S. (2018): Granitoidassociated gold mineralization in Egypt: A case study from the Atalla mine. Mineral. Deposita, 53, 701-720. 
بترولوجية وجيوكيميائية سرينتين أوفيوليتى بمنطقة أم عش، الصحراء الثرقية، مصر : وشاح نيويروتيروزوى من

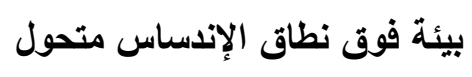
أيمن البدرى موريس

قسم الجيولوجيا - كلية العلوم - جامعة حلوان

الخلاصة

يشتنمل السـرنتنين الأوفيوليتى النيوبروتيروزوى لمنطقة أم عش بوسط الصـحراء الثـرقية المصـرية على أنواع كتليـة

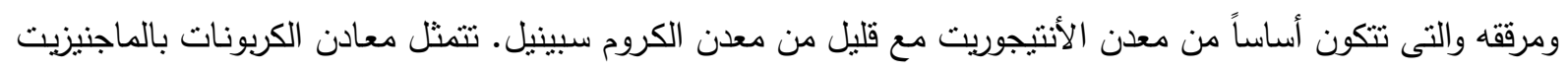

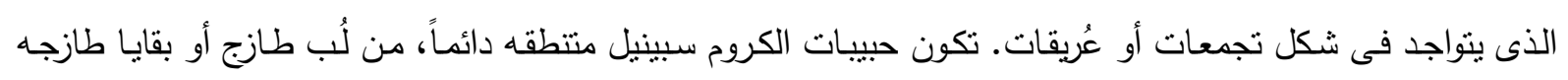

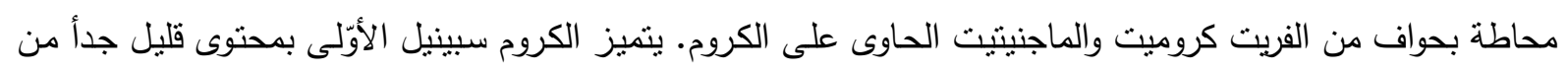

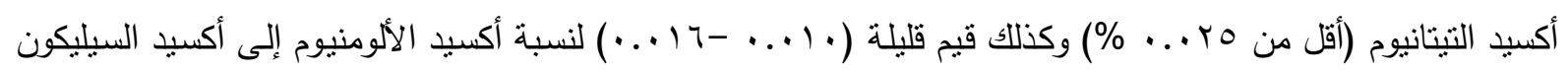
مما يوضح أن صخور السربنتين تمثل وشـاح متبقى تعرّض لدرجه عالية من الأنصهار الجزئى. ويتشابه محتوى الكروم

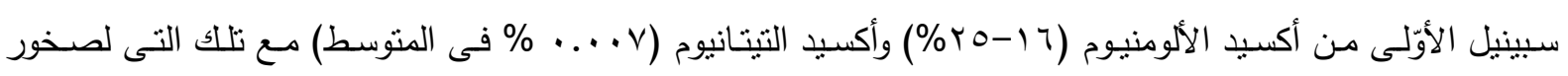

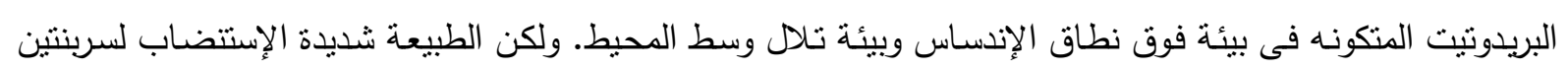

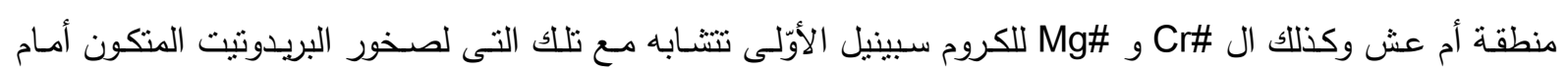
القوس لبيئة فوق نطاق الإندساس.

وأظهرت قطاعات للتركيب الكيميائى للنطاقات المختلفة للكروم سبينيل المتتطق أن تركيزات أكاسيد الكروم والمغنسيوم

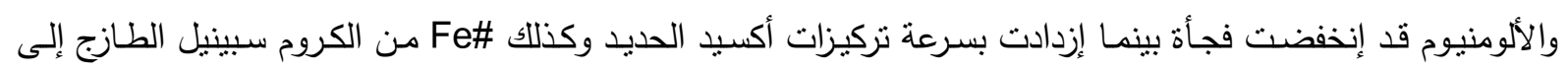

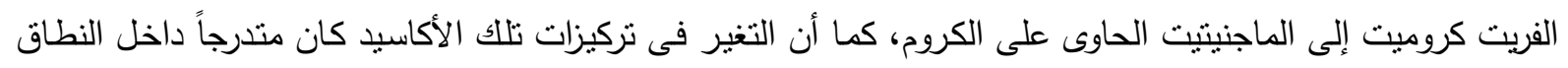
الواحد من نطاقات السبينيل المتحول. وعلى الجانب الأخر فأن نركيزات أكاسيد النيكل والمنجنيز تتغير بشكل غيرك فير منتظم

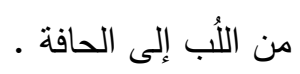

تؤكد سيادة معدن الأنتيجوريت فى صخور السربنتين ووجود حواف من السبينيل المتحول حول الكروم سبينيل الأوّلى أن صخور سربنتين أم عش قد تعرضت إلى تحول فى سحنة الأمفيبوليت السفلى فى ظروف بئئة مؤكسدة. 

Egyptian Journal of Geology, v. 63, 2019, p.191-213 